\title{
Synergistic and Competitive Adsorption of Hydrophilic Nanoparticles and Oil-Soluble Surfactants at the Oil-Water Interface
}

\author{
Joeri Smits, Rajendra P. Giri, Chen Shen, Diogo Mendonça, Bridget Murphy, Patrick Huber,
} Kurosch Rezwan, and Michael Maas*

Cite This: Langmuir 2021, 37, 5659-5672

Read Online

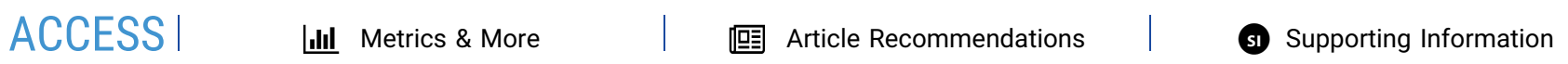

ABSTRACT: Fundamental insights into the interplay and self-assembly of nanoparticles and surface-active agents at the liquid-liquid interface play a pivotal role in understanding the ubiquitous colloidal systems present in our natural surroundings, including foods and aquatic life, and in the industry for emulsion stabilization, drug delivery, or enhanced oil recovery. Moreover, wellcontrolled model systems for mixed interfacial adsorption of nanoparticles and surfactants allow unprecedented insights into nonideal or contaminated particle-stabilized emulsions. Here, we investigate such a model system composed of hydrophilic, negatively, and positively charged silica nanoparticles and the oil-soluble cationic lipid octadecyl amine with in situ synchrotronbased X-ray reflectometry, which is analyzed and discussed jointly with dynamic interfacial tensiometry. Our results indicate that negatively charged silica nanoparticles only adsorb if the oil-water interface is covered with the

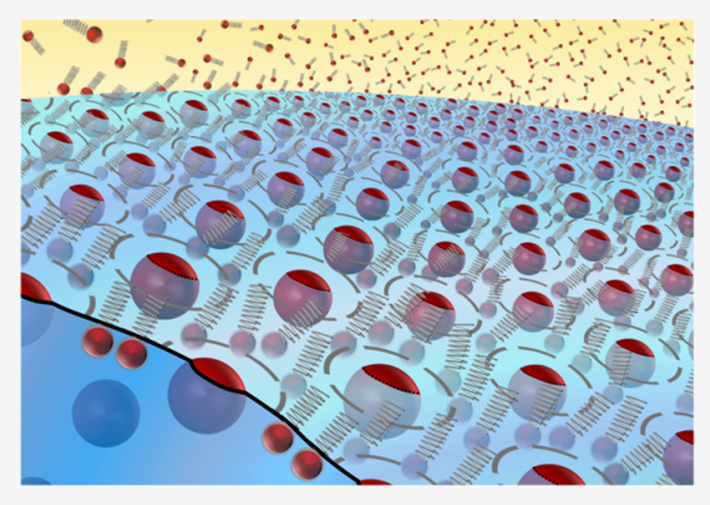
positively charged lipid, indicating synergistic adsorption. Conversely, the positively charged nanoparticles readily adsorb on their own, but compete with octadecyl amine and reversibly desorb with increasing concentrations of the lipid. These results further indicate that with competitive adsorption, an electrostatic exclusion zone exists around the adsorbed particles. This prevents the adsorption of lipid molecules in this area, leading to a decreased surface excess concentration of surfactants and unexpectedly high interfacial tension.

\section{INTRODUCTION}

Accurate determination of the psychochemical interfacial properties and interactions of mixed films combining nanoparticles (NPs) and surfactants adsorbed at a gas-liquid surface or liquid-liquid interface remains challenging. The long-standing debate is complicated by the limited available experimental and computational approaches along with large variability in particle materials (e.g., size, shape, wettability, etc.), ${ }^{1-5}$ the spectrum of environmental conditions (e.g., solvent, ${ }^{6-8} \mathrm{pH}^{9,10}$ ion concentration ${ }^{9,11}$ or the presence of surface-active impurities ${ }^{12}$ ), and the often unique interplay between sets of surfactants and particles.,13-15 Since surfactants allow tailoring the final structure, ${ }^{16}$ stability, ${ }^{17}$ or flow behavior ${ }^{18}$ of emulsions/foams decorated with particles, insights into the driving forces behind particle adsorption and desorption are critical for advances in the synthesis of emulsions and foams, ${ }^{16-18}$ froth floatation, ${ }^{19}$ drug encapsulation, ${ }^{14}$ and enhanced oil recovery. ${ }^{20}$

Based on the earlier findings of Pickering ${ }^{21}$ and Ramsden, ${ }^{22}$ Pieranski $^{23}$ observed irreversible adsorption of spherical polystyrene particles at the air-water interface and laid the groundwork for the well-known equation of lowering the surface free energy upon adsorption $\left(\Delta E_{P}\right)$ created by a decrease in the interfacial area by the particle of radius $(r)$ at equilibrium $^{24,25}$

$$
\Delta E_{\mathrm{P}}=-\pi r^{2} \gamma_{0}(1 \pm \cos \theta)^{2}
$$

where $\gamma_{0}$ is the pristine surface (SFT) or interfacial (IFT) tension and $\theta$ is the three-phase contact angle (CA) measured through the water phase. The sign between brackets is negative in case $\theta<90^{\circ}$ and positive when $\theta>90^{\circ}$ for a hydrophilic or hydrophobic particle, respectively. Determination of the equilibrium $\mathrm{CA}(\theta)$ for a NP trapped at an interface is derived from the Young-Dupré law ${ }^{26}$

$$
\cos \theta=\frac{\gamma_{\mathrm{po}}-\gamma_{\mathrm{pw}}}{\gamma_{\mathrm{wo}}}
$$

Received: February 26, 2021

Revised: April 8, 2021

Published: April 27, 2021 
Accordingly, the CA is determined by the interfacial tension between each phase, namely, particle-oil $\left(\gamma_{\mathrm{po}}\right)$, particle-water $\left(\gamma_{\mathrm{pw}}\right)$, and water-oil $\left(\gamma_{\mathrm{wo}}\right)$. Both equations describe the interfacial partitioning of a single particle at thermodynamic equilibrium. In reality, additional interfacial forces arise when multiple particles adsorb at the interface, influencing their adsorption energies and CAs, for example, DerjaguinLandau-Verwey-Overbeek (DLVO) interactions between the adsorbed particles or capillary interactions via line tension. ${ }^{27,28}$ Equation 1 has been utilized in axisymmetric droplet shape experiments to determine equilibrium adsorption energies and kinetics of surface-active nano- and micronsized particles based on the tentative assumption that a closepacked, densely populated particle film has formed when further increasing the bulk particle concentration results in constant SFT/IFT. ${ }^{2,3,11,29,30}$ However, the spatial confinement of the particles at the interface along with long-range repulsive electrostatic forces and attractive capillary interactions between adsorbed particles and between adsorbed particles with those in the bulk must be considered. This sets up an energy barrier for further adsorption and densification, which limits particle coverage well below what might be expected from a hexagonally close-packed (HCP) monolayer. ${ }^{1,31,32}$

In the case of interfacial systems with at least two surfaceactive species (e.g., particles and surfactants), the net energy balance between them dictates the final properties of the mixed interfacial film. Dispersing NPs with nonionic or oppositely charged surfactants in the same liquid medium generally modifies the particle hydrophobicity through hydrogenbonding and electrostatic interactions. ${ }^{33,34}$ In principle, particle-surfactant complexation explains differences in SFT/ IFT of mixed interfaces compared to particle-free interfaces, which are highly dependent on the concentration of each component. ${ }^{13,15,35-39}$ Complementary harmonic sinusoidal perturbations of pendant drops provide the complex dilational modulus that tends to increase for mixed films upon particle adsorption. ${ }^{15,40,41}$ In the case of equally charged surfactants and NPs dispersed in water, interactions are expected to be repulsive, but the exact mechanism determining the adsorption balance remains poorly understood. For example, the IFT and dilational moduli for silica NPs in the presence of negatively charged surfactants at the heptane-water interface have been attributed to the electrostatic repulsion between both species, ${ }^{42}$ while a recent study ascribes this difference to an increase in the surfactants surface activity induced by dissociation of the surface groups of the silica NPs, which increases the ionic strength of the bulk. ${ }^{43}$ Additionally, when particles and surfactants of opposite polarity are dispersed in different immiscible phases, their interactions are limited to the interfacial region. For example, Whitby et al. ${ }^{44}$ observed synergistic interactions between hydrophilic silica NPs and a cationic lipid (octadecyl amine, ODA) only when sufficient surfactant was adsorbed at the interface, in agreement with dilational rheology as indicated by an overall increase of the elasticity compared to the absence of NPs.

One major hurdle in estimating particle coverage or adsorption energies of adsorbed NPs is to predict their CA, which is mostly assumed from sessile droplets on chemically equivalent macroscopic substrates. Based on this tentative assumption, we recently showed that positively charged hydrophilic silica NPs reversibly adsorb in the presence of a cationic oil-soluble surfactant in a competitive manner. This conclusion was derived from a simple analytical model based on the principle of additivity of the interfacial energy contributions of the different components, ${ }^{45}$ which we used to assess the particle coverage in the absence and presence of NPs from IFT measurements ${ }^{46}$

$$
E_{\mathrm{i}}=\gamma_{\mathrm{SF}} A_{\mathrm{SF}}+N_{\mathrm{P}} \pi r^{2} \gamma_{\mathrm{ow}}(1-\cos \theta)^{2}
$$

where the total interfacial free energy $\left(E_{\mathrm{i}}\right)$ is dependent on the surfactant contribution (IFT with only surfactant $\left(\gamma_{\mathrm{SF}}\right)$ and the surfactant-covered fraction of the interface $\left.\left(A_{\mathrm{SF}}\right)\right)$ and of the adsorbed NPs (eq 1 at oil-water times the number of adsorbed NPs $\left.\left(N_{\mathrm{P}}\right)\right)$. Equation 3 is valid when particlesurfactant interactions are absent, meaning that the CA does not change upon the addition of ODA, and its derivation is described in the Methods section (eqs 4-9. Based on this assumption, the particle coverage of a mixed interfacial film can be derived by measuring the change in IFT when particles are added to the surfactant-laden interface.

For a more detailed analysis, and to validate our previous approach, tensiometry of mixed particle-surfactant dispersions needs to be bolstered by additional methods that can more directly obtain NP coverage and/or their three-phase CA at the oil-water interface. In this respect, several advanced microscopy methods for the observation of particles trapped at the interface have been developed, e.g., the gel trapping technique, ${ }^{47}$ solvent or gas polymerization, ${ }^{1,48}$ or freezefracture shadow-casting cryo-scanning electron microscopy (FreSCa cryo-SEM). ${ }^{49,50}$ Light scattering methods offer an alternative for studying adsorption at quiescent interfaces as an ensemble, and the determination of their properties involves a varying degree of modeling to obtain the $\mathrm{CA}^{51}$ and second harmonic generation ( $\mathrm{SHG}$ ) to estimate adsorption energies. ${ }^{52}$ For direct in situ analysis applicable to a wide range of interfacial systems, advanced synchrotron X-ray reflectivity (XRR) provides a detailed picture of a quiescent surface or buried interface with sub-Angstrom resolution perpendicular to the interface and averaged over the plane of the interface. Based on Parratt's recursive formalism, the backgroundcorrected reflectivity data can be modeled to obtain electron density (ED) profiles providing layer thickness, density, and roughness. Thus, it is possible to ultimately extract the interfacial composition with known material EDs. For evaluating particle adsorption, an additional computational step is required to extract information such as immersion depth and particle coverage by applying a physical model that directly correlates realistic properties of the interface and NPs. This approach was, for example, used by Calzolari et al. ${ }^{53}$ who investigated the adsorption of hexadecyltrimethylammonium bromide (CTAB)-coated silica NPs adsorbed at hexane-water using IFT combined with XRR. The same model and approach were later extended to polymer-decorated iron oxide NPs at the decane-water interface. ${ }^{54}$

Here, we utilize XRR in conjunction with tensiometry and dilational rheology to understand the adsorption and selfassembly of hydrophilic NPs in the presence of the cationic, oil-soluble surfactant octadecyl amine (ODA) at the decanewater interface. The particles have an equal or opposite charge to the ionic surfactant, which is dissolved in the immiscible oil phase to ensure their interactions are limited to the interfacial region. For this purpose, we use native negatively charged silica NPs and positively charged (3-aminopropyl)triethoxysilane (APTES)-coated silica NPs dispersed in the aqueous phase at $\mathrm{pH} 5.8$ at a fixed concentration of $0.1 \mathrm{w} / \mathrm{w} \%$. High purity of all 
constituents from further surface-active components is strictly observed during these experiments. The XRR data are analyzed via physical model fitting of the EDs adopted from Isa et al. ${ }^{54}$ NP coverage and CA obtained through modeling of the EDs are compared to and discussed with our previously reported continuum-based analytical model using the surface pressure difference in the presence and absence of APTES-coated silica NPs. ${ }^{46}$

\section{MATERIALS}

Tetraethyl orthosilicate (TEOS $\geq 99.0 \%$, product no. 86578), (3aminopropyl)triethoxysilane (APTES 99.0\%, product no. 440140), citric acid (anhydrous, $\geq 99.5 \%$, product no. 27109), potassium chloride ( $\mathrm{KCl} 99.0-100.5 \%$, product no. P3911), L-arginine ( $\geq 99.0 \%$, product no. 1.01542), octadecyl amine (ODA, $\geq 99.0 \%$, product no. 74750 ), and Hellma optical glass cuvettes (volume, $3.5 \mathrm{~mL} ; 45 \times 12.5$ $\times 12.5 \mathrm{~mm}^{3}$, product no. Z600008) were purchased from SigmaAldrich (Darmstadt, Germany). Decane ( $\geq 99.7 \%$, product no. D901), $\mathrm{HCl}(0.1 \mathrm{M}$, product no. 38280; $1 \mathrm{M}$, product no. 71763$)$, and $\mathrm{NaOH}$ (1 M, product no. 71463) solutions were purchased from Honeywell and $0.1 \mathrm{M} \mathrm{NaOH}$ (product no. 124200010) from Acros Organics (Nidderau, Germany). Acetone (99.0\%, product no. 20063.365), ethanol absolute ( $>99.8 \%$, product no. $64-17-5)$, and glacial acetic acid ( $\geq 99.7 \%$, product no. 64-19-7) were obtained from VWR Chemicals (Hannover, Deutschland). Technical-grade ethanol (99.0\%, product no. 2211.5000) was bought from Chemsolute (Hamburg, Germany). Kapton foil $\left(200 \times 304 \times 0.05 \mathrm{~mm}^{3}\right.$, product no. 536-3952) was obtained from RS Components GmbH (Frankfurt am Main, Germany), and ZelluTrans dialysis tubes (MWCO 6-8 $\mathrm{kDa}$, product no. E665.1) were obtained from Carl Roth $\mathrm{GmbH}$ (Karlsruhe, Germany). A Minisart syringe filter polyethersulfone (PES) with $0.1 \mu \mathrm{m}$ pore size (product no. 16553---------K) was obtained from Sartorius Stedim Biotech (Germany, Göttingen). All aqueous solutions were made with double-deionized water (18.2 $\mathrm{M} \Omega$ $\mathrm{cm}^{-1}$ at $25^{\circ} \mathrm{C}$ ) from a Synergy Water Purification system (Millipore Corp., Massachusetts).

\section{EXPERIMENTAL SECTION}

Generally, and well beyond the usual laboratory practice, great care was taken that all chemicals and final particle dispersion were of the highest purity and behaved chemically inert toward the used laboratory materials to ensure that all reported measurements are only caused by the described particles and lipid. ${ }^{12,55}$ All aqueous solutions were degassed prior to use.

Cleaning of Material. All glassware was cleaned by rinsing thrice with technical-grade ethanol and acetone, water, and ethanol absolute; covered with a lint-free wipe; and dried in air. If there was contact with surfactants, the glassware was first rinsed with technical-grade ethanol and with a soft brush cleaned in hot tap water with soap followed by copious rinsing with tap water, DEMI water, and Millipore water. Also, after using acetone, the material was rinsed with $6 \%$ citric acid. After the first rinse with citric acid, the glassware was filled with the acidic solution and placed in an oven for $30 \mathrm{~min}$ at 70 ${ }^{\circ} \mathrm{C}$ before continuing with water and ethanol.

Nanoparticle Synthesis. Silica and APTES-coated silica nanoparticles were synthesized and functionalized in a one-pot reaction. A concentrated, alcoholic prehydrolyzed solution of APTES (1:0.95:0.05 ethanol absolute/APTES/water, v/v\%) was prepared 1 day in advance and stored at $4{ }^{\circ} \mathrm{C} .{ }^{56}$ First, $300 \mathrm{mg}$ of L-arginine was magnetically stirred $(40 \times 10 \mathrm{~mm})$ with $300 \mathrm{~mL}$ of water in a $500 \mathrm{~mL}$ round-bottom flask at $70^{\circ} \mathrm{C}$ with reflux for at least $1 \mathrm{~h}$. Afterward, 32 $\mathrm{mL}$ of TEOS was added dropwise at $20 \mathrm{~mL} \mathrm{~h}^{-1}$ with a stirring speed of $1000 \mathrm{rpm}$, and this speed was maintained for $1 \mathrm{~h}$ after the addition of TEOS. The stirring was stopped for $15 \mathrm{~min}$, allowing TEOS to phase-separate, and stirring was continued for at least $24 \mathrm{~h}$ at 250 $\mathrm{rpm}$. When all TEOS was consumed, the silica particles were heated to $90{ }^{\circ} \mathrm{C}$ at $750 \mathrm{rpm}$ for $24 \mathrm{~h}$. For coating with APTES, the synthesized particles were cooled down in an ice bath to approximately $4-6{ }^{\circ} \mathrm{C}$. The $\mathrm{pH}$ was lowered to approximately 5 by dropping $100 \mathrm{mM}$ acetic acid diluted in ethanol absolute at $40 \mathrm{~mL} \mathrm{~h}^{-1}$ with high stirring, so the final solution had a concentration of $10 \mathrm{v} / \mathrm{v} \%$ of ethanol and was continued for $30 \mathrm{~min}$ post addition. The prehydrolyzed APTES solution was allowed to thermally equilibrate to room temperature, while the particle dispersion was reheated to 40 ${ }^{\circ} \mathrm{C}$ with reflux. Prehydrolyzed APTES $(20 \mathrm{~mL})$ was added at $10 \mathrm{~mL}$ $\mathrm{h}^{-1}$ with stirring at $1000 \mathrm{rpm}$. Afterward, the temperature was increased to $90{ }^{\circ} \mathrm{C}$ for $24 \mathrm{~h}$ with stirring at $750 \mathrm{rpm}$. The final $\mathrm{pH}$ increased to 8-9, and another $100 \mathrm{mM}$ acetic acid dissolved in $5 \mathrm{~mL}$ of ethanol absolute was added dropwise manually until the final $\mathrm{pH}$ was 5. Particles were stored at $4{ }^{\circ} \mathrm{C}$ or cooled down before purification.

Nanoparticle Purification. The silica dispersion was first filtered (PES $0.1 \mu \mathrm{m}$ ) and APTES-coated silica particles were centrifuged at $5000 \mathrm{rpm}$ for $30 \mathrm{~min}$ to remove large agglomerates. Two dialysis bags, each containing $30 \mathrm{~mL}$ of the particle dispersion were placed in a $2 \mathrm{~L}$ beaker containing 1:0.5:0.007 of water/ethanol absolute/acetic acid for at least $4 \mathrm{~h}$ and repeated twice. The particles were 10 times dialyzed against water $\mathrm{pH} 5.8$ with a minimum time interval of $4 \mathrm{~h}$. Upon completion of the dialysis, water was refreshed one final time and stirred for $30 \mathrm{~min}$ before collecting the particles in a clean Duran beaker with a PTFE-coated cap. The $\mathrm{pH}$ was adjusted to 5.8 if necessary, and the solid content was determined in triplicate to be $2.32 \pm 0.02$ for silica and $1.76 \pm 0.04 \mathrm{w} / \mathrm{w} \%$ for APTES-coated silica and stored at $4{ }^{\circ} \mathrm{C}$. Both dialyzed dispersions remained stable for at least 3 months.

Nanoparticle Characterization. Transmission electron microscopy (TEM-EM 900, Carl Zeiss, Germany) was employed to investigate the morphology of the particles by placing a $3 \mu \mathrm{L}$ water drop of $0.001 \mathrm{w} / \mathrm{w} \%$ particle dispersion $(\mathrm{pH} \sim 5.8)$ onto a copper grid (Plano GmbH, Wetzlar, Germany). Size and $\zeta$ potential were obtained by measuring $0.1 \mathrm{w} / \mathrm{w} \%$ particle dispersion in $10 \mathrm{mM} \mathrm{KCl}$ for conductivity at different $\mathrm{pH}$ values using the ZetaSizer NanoSP (Malvern, United Kingdom) at $25{ }^{\circ} \mathrm{C}$. To remove all organic impurities, $20 \mathrm{~g}$ of $\mathrm{KCl}$ was dissolved in $200 \mathrm{~mL}$ of water and heated to $550{ }^{\circ} \mathrm{C}$ for $24 \mathrm{~h}$. The $\mathrm{pH}$ of the particles was adjusted by adding 0.1 or $1 \mathrm{M} \mathrm{KOH}$ and $\mathrm{HCl}$ and stirred for 15 min followed by another $\mathrm{pH}$ reading right before measuring the $\zeta$ potential. The expression proposed by Ohshima et al. ${ }^{57}$ was used to calculate the Henry function $f\left(\begin{array}{ll}\kappa & a\end{array}\right)$ by implementing the hydrodynamic radius and electrolyte concentration. The calculated $f(\kappa a)$ values were 1.11 and 1.13 for silica and APTES-coated silica, respectively.

Ninhydrin Assay. Primary amine quantification of APTES-coated silica particles was adopted from Hristov et al. ${ }^{58}$ A calibration curve was obtained by dissolving 1-3 $\mathrm{mM}$ APTES in 1:0.027 ethanol absolute:acetic acid (Figure S1, Supporting Information). The purified particles were dialyzed twice against the ethanol absolute:acetic acid mixture and diluted to $0.05 \mathrm{w} / \mathrm{w} \%$. A ninhydrin $(20 \mathrm{mM})$ stock solution was prepared fresh in the dark. To a Safe Lock Eppendorf tube, $0.125 \mathrm{~mL}$ of ninhydrin was mixed with $0.375 \mathrm{~mL}$ of the calibration solution or particle dispersion. A control sample without ninhydrin and particles was also prepared and used as baseline subtraction for the reacted samples. The tubes were incubated in the dark for $45 \mathrm{~min}$ at $60{ }^{\circ} \mathrm{C}$, whereafter $200 \mu \mathrm{L}$ of the suspensions was pipetted into a 96-well plate. A maximum absorption occurred at a wavelength of $577 \mathrm{~nm}$, and samples were measured in triplicate. For calculating the amine density per $\mathrm{nm}^{2}$, assumptions were made that all particles are perfect spheres of the same size $(18.9 \mathrm{~nm})$ and their density is equal to $1.9 \mathrm{~g} \mathrm{~cm}^{-3}$. 59

Preparation of Lipids in Decane. A stock solution of $4 \mathrm{mM}$ ODA was prepared 4 days in advance of the synchrotron experiments at room temperature to ensure a homogeneous dissolution of the lipid and was also used afterward for the pendant drop experiments.

Pendant Drop Tensiometry and Dilational Interfacial Rheology. Tensiometry and dilational rheology of the oil-water interface was studied at $20{ }^{\circ} \mathrm{C}\left( \pm 1{ }^{\circ} \mathrm{C}\right)$ using the OCA20 from Dataphysics (Stuttgart, Germany) with a sensitivity of $0.01 \mathrm{mN}$ $\mathrm{m}^{-1} .{ }^{\circ}$ A $20-25 \mu \mathrm{L}$ aqueous drop $(\mathrm{pH} \sim 5.8)$ was formed from a dosing needle (1.07 mm diameter) in a cuvette filled with $900 \mu \mathrm{L}$ of 


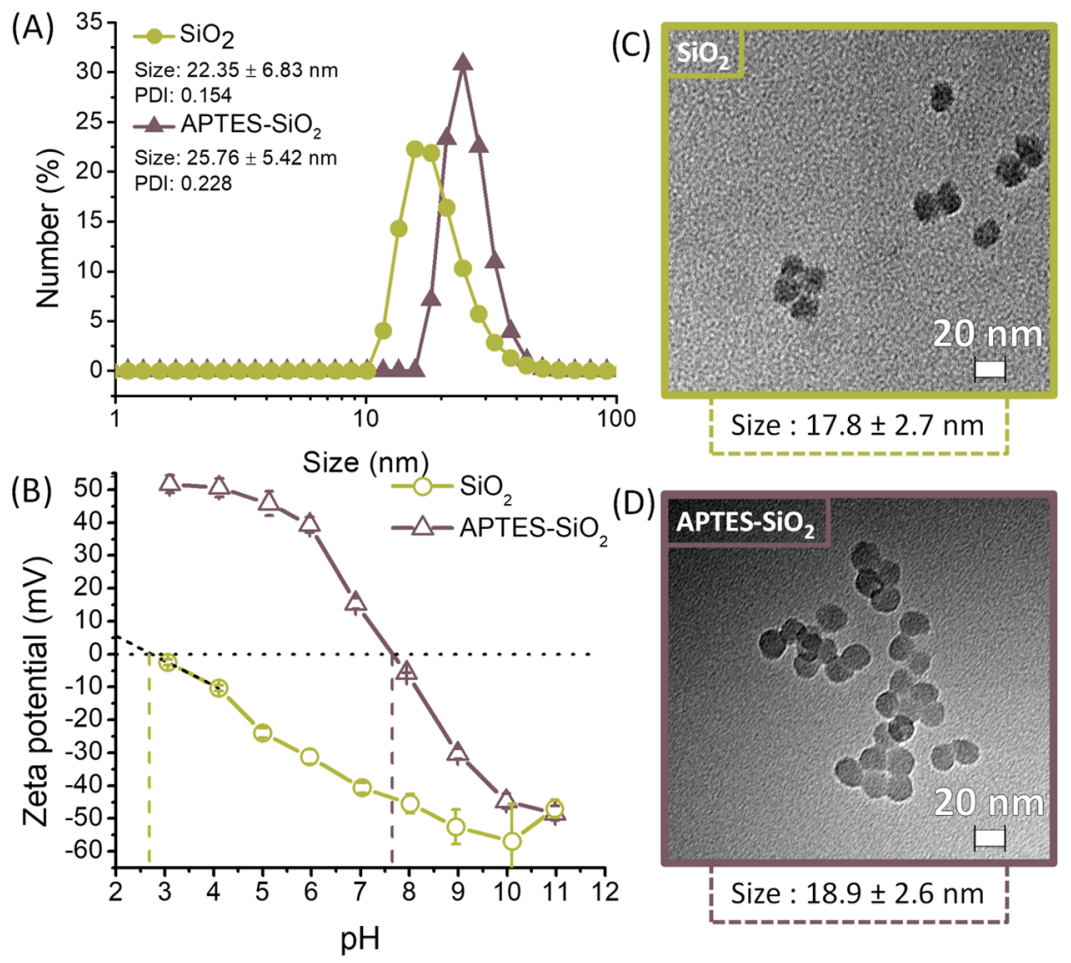

Figure 1. (A) Hydrodynamic diameter and polydispersity of silica (circles) and APTES-coated silica (triangles) nanoparticles. (B) $\zeta$ potential of 0.1 $\mathrm{w} / \mathrm{w} \%$ silica and APTES-coated silica as a function of $\mathrm{pH}$ in $10 \mathrm{mM} \mathrm{KCl}$. Isoelectric points (IEPs) are indicated by the dashed line for clarity. The IEP of silica was extrapolated in OriginPro8. Transmission electron microscopy images of silica (C) and APTES-coated silica (D) showing size and standard deviations.

pure decane to check the purity if the tension remained constant with a systematic error of $0.2 \mathrm{mN} \mathrm{m}^{-1}$. This is equally true for the aqueous phase regarding the purity of particle dispersions (Figure S3A,B, Supporting Information). Before the addition of lipids, the tension was monitored for $30 \mathrm{~min}$, after which $900 \mu \mathrm{L}$ of the required lipid concentration was slowly added to avoid significant droplet disturbance and measured for $2 \mathrm{~h}$. Dilational viscoelastic properties of the drops were obtained by sinusoidal volume oscillations (ODG25, Dataphysics) at varying frequencies between 0.005 and $0.2 \mathrm{~Hz}$ at a fixed area deformation of $9-10 \%$ after the tensiometry test. Each system was measured at least three times, and the experimental error is reported (Figure S3C, Supporting Information).

Analytical Model. The analytical model is a continuum-based approach considering additivity of interfacial free energy $\left(E_{\mathrm{i}}\right)$ contributions of ODA $\left(E_{\mathrm{SF}}\right)$ and adsorbed $80 \mathrm{~nm}$ APTES-coated silica NPs $\left(N_{\mathrm{P}} \Delta E_{\mathrm{P}}\right)$. The difference in IFT in the presence and absence of NPs at different ODA concentrations allows estimating the particle coverage. ${ }^{46}$

$$
E_{\mathrm{i}}=\gamma_{\mathrm{SF}} A_{\mathrm{SF}}+N_{\mathrm{P}} \pi r^{2} \gamma_{\mathrm{ow}}(1-\cos \theta)^{2}
$$

which can be rewritten as

$$
E_{\mathrm{i}}=E_{\mathrm{SF}}+N_{\mathrm{P}} \Delta E_{\mathrm{P}}
$$

where the first term is the product of the equilibrium IFT in the presence of only surfactant $\left(\gamma_{\mathrm{SF}}\right)$ and the surfactant-covered fraction of the interface $\left(A_{\mathrm{SF}}\right)$, and the right term is eq 1 times the number of NPs $\left(N_{\mathrm{P}}\right)$. This is valid when particle-surfactant interactions are absent, meaning that the CA does not change upon the addition of ODA. The total interfacial free energy $\left(E_{\mathrm{i}}\right)$ is related to the apparent IFT $\left(\gamma_{\mathrm{i}}\right)$ and total interfacial area $\left(A_{\mathrm{i}}\right)$ and is expressed as

$$
\gamma_{\mathrm{i}}=\frac{E_{\mathrm{i}}}{A_{\mathrm{i}}}
$$

Therefore, in the absence of particle-surfactant interactions, the difference in IFT (or just surface pressure) indirectly accounts for the number of adsorbed particles. The IFT from the pendant drop (with known droplet area) corresponds to the free energy difference with and without NPs $\left(\Delta E_{\mathrm{i}}\right)$. Contact angle measurements allowed us to estimate the adsorption energy of one $80 \mathrm{~nm}$ APTES-coated silica NP and of several ODA molecules that would normally occupy the same area as one $\mathrm{NP}\left(\Delta E_{\mathrm{Ads}}\right)$.

From this follows

$$
\frac{\Delta E_{\mathrm{i}}}{\Delta E_{\mathrm{Ads}}}=N_{\mathrm{P}}
$$

If the CA of one NP is known, its occupational interfacial area $\left(A_{\mathrm{P}}\right)$ can be calculated and the maximum number of adsorbed NPs $\left(N_{\max }\right)$ can be derived from the experimental droplet volume $\left(A_{\mathrm{i}}\right)$ and the hexagonal close-packing factor

$$
\frac{A_{\mathrm{i}}}{A_{\mathrm{P}}} 0.91=N_{\max }
$$

This allows estimating the particle coverage

$$
\frac{N_{\mathrm{P}}}{N_{\max }}=\varphi
$$

X-ray Reflectivity. Experimental Setup. The reflectivity measurements (XRR) at the decane-water interface were performed at beamline P08 at PETRA III for its delivery of X-rays with high brilliance (DESY, Hamburg, Germany), using the liquid interface diffractometer LISA. ${ }^{61,62}$ The $25 \mathrm{keV}$ beam at the collimated mode allows penetration through the decane super phase and probes the buried decane-water interface with a beam size of $0.05 \times 0.4 \mathrm{~mm}^{2}(V$ $\times H)$. During the XRR measurement, the incident angle concerning the liquid surface or liquid-liquid interface was varied using the LISA double-crystal beam tilter to obtain a reflectivity profile as a function of the momentum transfer $q=4 \pi \sin \left(\theta_{\mathrm{i}}\right) / \lambda$, where $\lambda=0.496 \AA$ (Figure S4, Supporting Information).

The sample was prepared in a custom-made cell consisting of an aluminum mounting cell hosting a Delrin cup (inner diameter, 60 

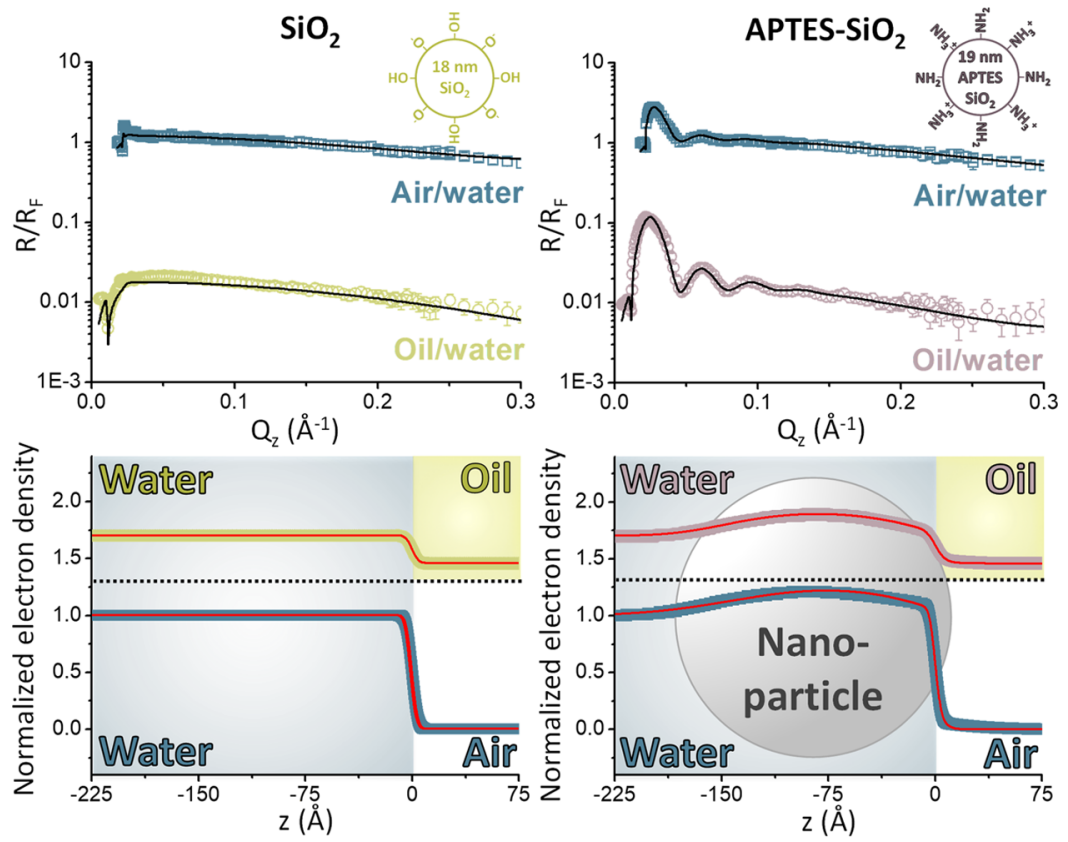

Figure 2. (Top row) Normalized reflectivity $R / R_{\mathrm{F}}$ data along with the fitted line (black) for $0.1 \mathrm{w} / \mathrm{w} \%$ silica (left) and APTES-coated silica (right) at the air-water (squares) and decane-water (circles) interface (aqueous $\mathrm{pH}$ 5.8). Data are shifted by a factor of 100 for clarity. (Bottom row) Stacked normalized ED curves with the model fits (red) with a corresponding schematic in the background.

$\mathrm{mm}$ ) as shown in Figure S5 (Supporting Information) with more detailed information. The cell was mounted on a goniometer to be positioned perfectly horizontal and filled with $28 \mathrm{~mL}$ of $0.1 \mathrm{w} / \mathrm{w} \%$ particle dispersion $(\mathrm{pH} \sim 5.8)$ to pin the meniscus and create a flat interface. Decane $(20 \mathrm{~mL})$ was added slowly via the sides of the Delrin cup. The amount of decane was roughly $1 \mathrm{~cm}$ thick for the incident and reflected X-rays to traverse only through the decane phase, which eliminates scattering of the decane-air interface. After a clean decane-water interface was established, a calculated amount of decane was removed and replaced with an equal amount of $4 \mathrm{mM}$ ODA stock solution to keep the oil volume constant at $20 \mathrm{~mL}$. The ODA concentration was varied from 0.01 to $1 \mathrm{mM}$ for each set of measurements, and several reflectivity curves were recorded for each concentration, which showed only slight variations and no trend. REFLEX software based on Parratt's recursive algorithm was used to analyze the reflectivity curves with known material electron density (ED) of the different layers (Figure S2, Tables S1 and S2 in the Supporting Information). ${ }^{63}$ The ED for silica was kept within physical boundaries determined from a mass density of $1.9 \mathrm{~g} \mathrm{~cm}^{-3,59}$ although higher densities are reported for macroscopic silica. ${ }^{53,58}$ Most of the parameters for the particle were highly constrained while allowing the material ED to vary. For clarity, the measured data and their fits were all normalized by the Fresnel reflectivity $\left(R_{\mathrm{F}}\right)$ of the corresponding interfaces in this paper.

Physical Model for Interfacially Adsorbed Nanoparticles. The physical model used here was adopted from Isa et al. ${ }^{54}$ The fitting of the experimental reflectivity data involves a Fourier transform that translates the data into an averaged electron density profile $\rho(\mathrm{z})$ within the plane of the reflecting interface to the surface normal. This electron density profile is then fitted to a model that takes into account the physical parameters of the interface and the shape of the NPs

$$
\begin{aligned}
\rho(z)= & \rho_{\mathrm{i}}(z)+\iint\left[\rho_{\mathrm{NP}}\left(s_{\mathrm{av}}, \rho_{\mathrm{p}} ; z\right)-\rho_{\mathrm{NP}}\left(s_{\mathrm{av}}, \rho_{\mathrm{i}}(z) ; z\right)\right] \\
& G\left(r_{\mathrm{av}}, \sigma_{\mathrm{r}} ; r\right) G\left(h_{\mathrm{av}}, \sigma_{\mathrm{h}} ; h\right) \mathrm{d} r \mathrm{~d} h
\end{aligned}
$$

The bare decane-water interface is described by $\rho_{\mathrm{i}}(\mathrm{z})$ with bulk electron densities (EDs) of water $\rho_{\mathrm{w}}=0.334 \mathrm{e} \AA^{-3}$ and decane $\rho_{\mathrm{d}}=$ $0.253 \mathrm{e} \AA^{-3}$ and an interfacial roughness $\sigma_{i}$ described by a Gaussian error function

$$
\rho_{\mathrm{i}}(z)=\rho_{\mathrm{w}}+\frac{\rho_{\mathrm{d}}-\rho_{\mathrm{w}}}{2}\left[1+\operatorname{erf}\left(\frac{z}{\sigma_{\mathrm{i}} \sqrt{2}}\right)\right]
$$

The contribution of the NP adsorbed at the interface is represented by $\rho_{\mathrm{NP}}(\mathrm{z})$, which is modeled as a monolayer of hexagonally packed spheres of radius $r$, immersion depth $h$, average interparticle spacing $s_{\mathrm{av}}$, and bulk particle electron density $\rho_{\mathrm{p}}$ (Figure 1)

$$
\begin{aligned}
\rho_{\mathrm{NP}}(z) & =\rho_{\mathrm{NP}}\left(s_{\mathrm{av}}, \rho_{\mathrm{p}} ; z\right) \\
& =\frac{2 \pi \rho_{\mathrm{p}}}{\sqrt{3}\left(2 r+s_{\mathrm{av}}\right)^{2}}\left[-z^{2}+2(r-h) z+h(2 r-h)\right]
\end{aligned}
$$

when $-h<z<-h+2 r$ and $\rho_{\mathrm{NP}}(z)=0$ otherwise. The subtracted term between brackets for eq 10 accounts for the liquid displacement by the NPs. Normalized Gaussian distributions $G\left(x_{\mathrm{av}}, \sigma_{x} ; x\right)$ (eq 10) are introduced to account for size polydispersity $(r)$ and consequently varying immersion depths $(h)$ with their average values $x_{\mathrm{av}}$ and their widths $\sigma_{x}$. All parameters were fixed to their known values or constrained except the average immersion depth $\left(h_{\mathrm{av}}\right)$ and the average separation distance between the NPs $\left(s_{\text {av }}\right)$. The former determines the particle contact angle and the $z$-coordinate of the maximum in the ED profiles, while the latter describes the average particle coverage associated with the height of the $\rho(z)$ peak and is schematically shown in Figure S4, Supporting Information.

\section{RESULTS AND DISCUSSION}

To obtain well-defined and highly pure particles, bare silica NPs were prepared via the Stöber-Watanabe process with the silane tetraethyl orthosilicate (TEOS) ${ }^{64}$ and alternatively coated with the silane (3-aminopropyl)triethoxysilane (APTES) to decorate the particle surface with amino groups, which is similar to the polar head group of ODA. The $p K_{a}$ of ODA is 10.65 , meaning that under the investigated environmental conditions, the protonated form dominates at the interface. ${ }^{65}$ Figure $1 \mathrm{~A}$ shows the number distribution of the silica and APTES-coated silica particles with hydrodynamic diameters of $22.35 \pm 6.83$ and $25.76 \pm 5.42 \mathrm{~nm}$, respectively. 

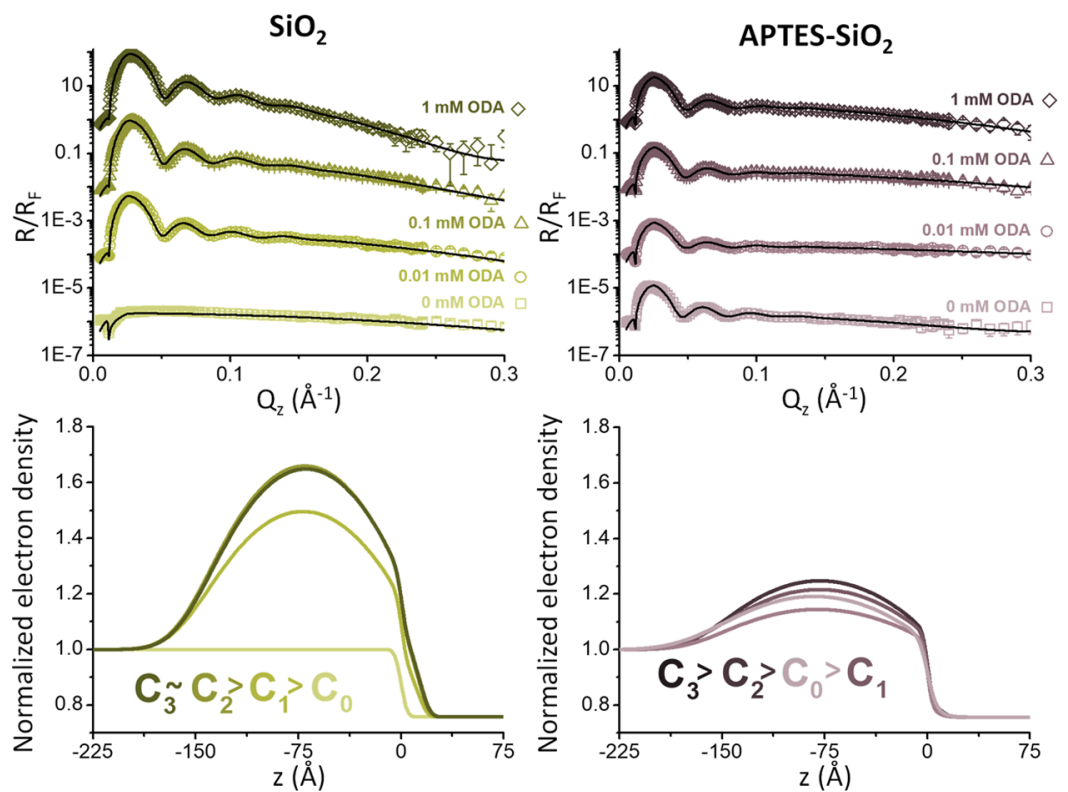

Figure 3. (Top row) $R / R_{\mathrm{F}}$ profiles of $0.1 \mathrm{w} / \mathrm{w} \%$ silica (left) and APTES-coated silica (right) at $\mathrm{pH} 5.8$ for different concentrations of ODA dissolved in the decane phase. Data are shifted by a factor of 100 for clarity. The normalized ED profiles are shown below where the peak amplitude is indicated in descending order. $\mathrm{C}_{0}$ means no surfactant is added, while the subscripts 1 to 3 correspond to increasing lipid addition from 0.01 to 1 mM ODA.

Both solutions exhibit low polydispersity with a polydispersity index (PDI) of 0.154 for silica and 0.228 for APTES-coated silica. Their isoelectric points are approximately 2.8 for silica and 7.6 for APTES-coated silica, confirming that both NPs are highly charged at the investigated $\mathrm{pH}$ of 5.8 and provide sufficient electrostatic stabilization to prevent particle-particle interactions in the bulk (Figure 1B). Approximately one amine group per $\mathrm{nm}^{2}$ is present on the particle surface determined with the ninhydrin assay (Figure S1, Supporting Information). ${ }^{58}$ Transmission electron microscopy (TEM) in Figure 1 shows that both silica (C) and APTES-coated silica (D) are nearly spherical with average diameters of $17.8 \pm 2.7$ and 18.9 $\pm 2.6 \mathrm{~nm}$, respectively.

Adsorption of highly hydrophilic, small NPs to a clean surface or interface is considered unfavorable and unstable due to their low adsorption energies. Particularly, XRR provides a highly detailed picture of the liquid interface and adsorbed materials. The top row of Figure 2 shows the reflectance profiles normalized by the theoretical Fresnel reflectivity $R_{\mathrm{F}}$ of $0.1 \mathrm{w} / \mathrm{w} \%$ silica (left) and APTES-coated silica particles (right) at the air-water and decane-water interface in the absence of ODA with their corresponding fits obtained with the standalone REFLEX software that simulates the XRR measurements based on Parratt's recursive formalism using a three-slab model (Figure S2, Supporting Information). ${ }^{63}$ The model parameters can be found in Table S1 (Supporting Information). The bottom row of Figure 2 shows the corresponding EDs, which are fitted using the physical model detailed in the Methods section. In the presence of silica NPs, both air-water and oilwater reflectivity curves are decaying monotonically as a function of $Q_{z}$ and resemble the Fresnel reflectivity with an interfacial roughness of pure water, ${ }^{66}$ indicating the absence of interfacial NP layering at $0.1 \mathrm{w} / \mathrm{w} \%$. This situation is clearly different for the APTES-coated silica particles, which are similar in size to the silica NPs but exhibit a positive $\zeta$ potential. Kiessig fringes appear at both air-water and oilwater interfaces from which the thickness $(d)$ can be estimated as $d=2 \pi / \Delta Q_{z}$ and is $18.74 \pm 1.45 \mathrm{~nm}$, consistent with the particle size determined from TEM (Figure 1D). Subsequent curve fitting of the reflectivity using the three-slab model confirms the monolayer formation (Figure 2, bottom). The normalized ED profiles for these adsorbing positively charged NPs show that most of their volume remains dispersed in the water phase owing to their hydrophilic character. Besides minimization of the free energy of the interface, the negative charge of a clean surface electrostatically attracts the positively charged NPs to the surface as observed by Larson-Smith et al., ${ }^{67}$ while it prevents adsorption of the negatively charged NPs. ${ }^{53,67}$ The origin of this negative charge can either be due to selective adsorption and interfacial ordering of hydroxyl ions at the boundary layer ${ }^{68-70}$ or from charge transfer between different interfacial water molecules. ${ }^{71}$

If the charge on the NP surface influences the adsorption based on electrostatics, one can expect that addition of an ionic surfactant will evoke either synergistic or competitive adsorption due to attractive and repulsive interfacial interactions, respectively. Figure 3 shows the $R / R_{\mathrm{F}}$ data and normalized EDs for both particle types in the presence of diverse quantities of ODA dissolved in the oil phase and the fit parameters for the reflectivity are shown in Table S2 (Supporting Information). Transitioning of the oil-soluble surfactant octadecyl amine (ODA) across the interface is insignificant at an aqueous subphase $\mathrm{pH}$ of 5.8, assuring particle-surfactant interactions occur solely at the phase boundary layer. ${ }^{65}$ The ED of ODA lies between that of water and decane, and the low scattering contrast makes it difficult to confirm its exact location and structuring, especially in the presence of the stronger scattering NPs. Noticeable oscillations appear for the negatively charged silica NPs when a small amount of $0.01 \mathrm{mM}$ ODA is added to the oil phase, assigned to electrostatic physisorption of the protonated amine group of ODA with the silanol groups on the particle surface similar to $\mathrm{CTAB}$-silica complexes. ${ }^{53}$ Furthermore, the surface potential of the interface might change to a positive value upon 

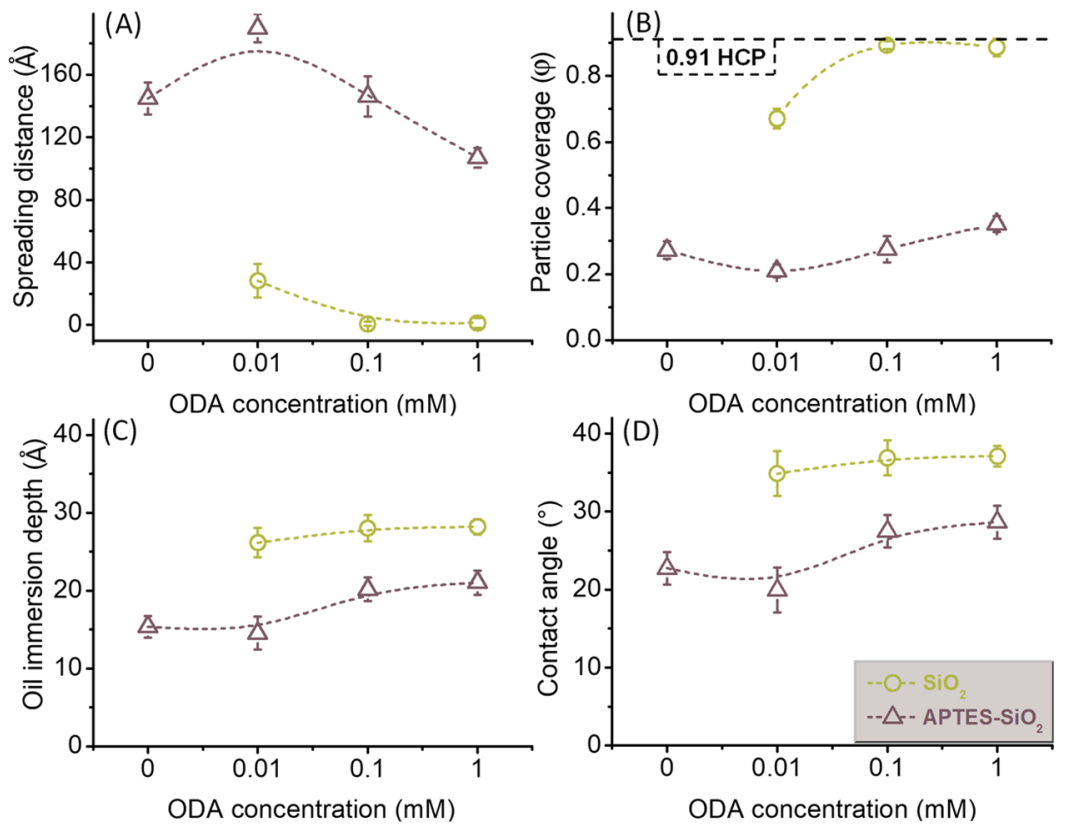

Figure 4. Results of the physical model for $0.1 \mathrm{w} / \mathrm{w} \%$ silica (circles) and APTES-coated silica (triangles) with or without ODA. Dashed lines are a guide to the eye. (A) Spreading distance between interfacially adsorbed particles, (B) packing density of adsorbed particles with the straight dashed line corresponding to hexagonal close-packing (HCP), (C) their protrusion distance into the oil phase, and (D) their three-phase contact angle, including standard deviations.

adsorption of ODA, also facilitating silica adsorption. A maximum in the amplitude of the ED curves is already achieved at $0.1 \mathrm{mM}$ ODA and a further increase in ODA concentration produces indistinguishable ED profiles. For the APTES-coated NPs, having roughly 1 amine group per $\mathrm{nm}^{2}$ on their surface (Figure S1, Supporting Information), a distinct pattern can be observed from the normalized reflectivity and EDs. After the addition of the lowest concentration $(0.01 \mathrm{mM}$ ODA), the oscillations are dampened compared to APTEScoated silica at the pure oil-water interface, and this decrease in intensity is caused by a smaller number of NPs at the interface. This confirms that particles and surfactants carrying a similar charge are competing at the interface for adsorption and the surfactant molecules can overpower the particles based on the repulsion between like charges, in line with our previous study. $^{46}$

Subsequently, the ED profiles are fitted to the physical model and the simulated parameters for particle diameter, average separation between neighboring particles, immersion depth, and CA (which is directly calculated from the immersion depth) are shown in Figure 4. The particle size from these fits estimated a diameter of $17.85 \pm 0.80 \mathrm{~nm}$ for silica and $18.93 \pm 1.14 \mathrm{~nm}$ for APTES-coated silica, ruling out particle multilayer formation at the interface for all ODA concentrations. The particle coverage was derived from the maximum amplitude of the ED as the ratio of the sample to the maximum silica NP density multiplied by the HCP factor $(\varphi=$ $\left.\left(\rho_{\text {sample }} / \rho_{\text {silica }}\right) 90.69\right)$. Figure $4 \mathrm{~A}$ shows that the spreading distance for 0.1 and $1 \mathrm{mM}$ ODA lies around zero and accordingly a coverage close to $100 \%$ in relation to $\mathrm{HCP}$, which corresponds to a densely packed particle monolayer. At $0.01 \mathrm{mM}$ ODA, already $73.98 \%$ of the interface is covered with silica NPs and the monolayer is saturated from $0.1 \mathrm{mM}$ ODA onward. The depth of immersion of the silica particles into the oil phase shows no significant difference between all ODA concentrations, with a CA between 34.9 to $37.1^{\circ}$. The results also suggest that, in the analyzed range, the surfactant concentration has a minor effect on the CA of the NPs irrespective of their coverage.

With the positively charged, aminated NPs and ODA, an opposite behavior can be expected compared to silica, especially since the NPs are spontaneously adsorbing at the pristine oil-water interface. Figure 4A shows an increase in the average particle-to-particle distance with the addition of 0.01 mM ODA. Gradually, the particles come closer when more lipids are added but remain separated from each other by $\sim 11$ $\mathrm{nm}$ at the highest concentration with only $38.75 \%$ coverage in relation to $\mathrm{HCP}$ (Figure 4B) and remain more deeply immersed in the water phase compared to the partially hydrophobized silica particles (Figure 4C). The initial CA of the APTES-coated particles in the absence of lipids $\left(\sim 22.3^{\circ}\right)$ acts as a reference to evaluate whether interactions occur between the particles and the equally charged surfactants. Previously, static oil-water CA measurements of oil-immersed water droplets on APTES-coated silica thin films on macroscopic surfaces showed no change in CA up to $1 \mathrm{mM}$ ODA despite having a higher initial CA of $40.05\left( \pm 1.43^{\circ}\right)$ that can be ascribed to the different synthesis process used. ${ }^{46}$ Figure 4D shows a gradual increase to $\sim 28.7^{\circ}$, and the difference of roughly $10^{\circ}$ in the wetting angle between the differently charged NPs shows that synergistic adsorption dominates with oppositely charged species while similarly charged species compete for interfacial coverage, suggesting fewer particlesurfactant interactions occur.

Adsorption studies using axisymmetric droplet shape analysis of a quiescent or perturbed oil-water interface provide complementary information to the specular reflectivity data by analyzing the time-resolved interfacial tension (IFT) in conjunction with frequency-dependent dilational rheology. The IFT of the pristine oil-water interface in the absence of NPs and for both negatively and positively charged NPs is constant, indicating the absence of surface-active impurities 


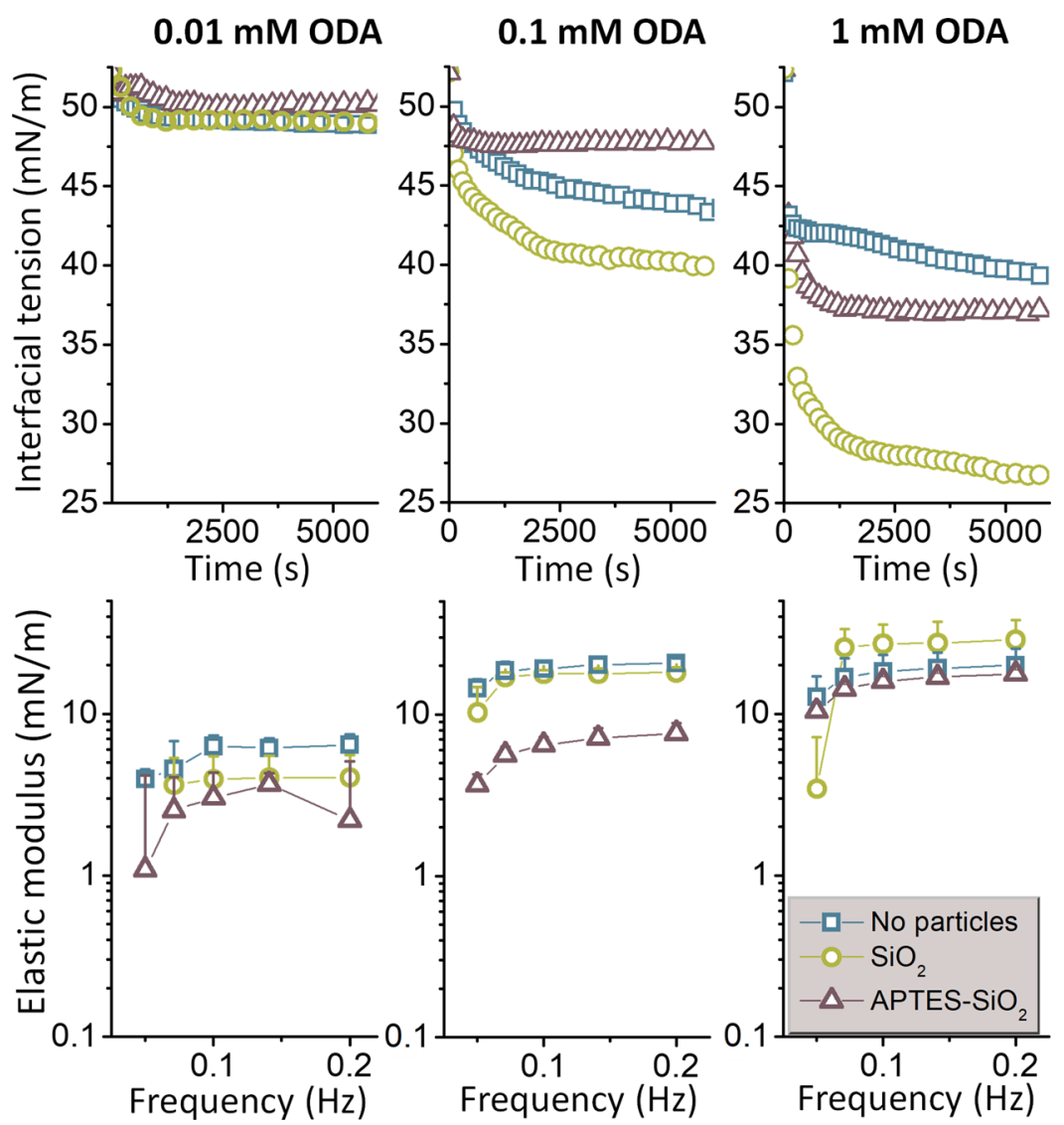

Figure 5. Data for various ODA concentrations measured by adsorption studies using axisymmetric droplet shape analysis. (Top row) Waterdecane interfacial tension without particles (squares) and with $0.1 \mathrm{w} / \mathrm{w} \%$ silica (circles) or APTES-coated silica (triangles) nanoparticles at $\mathrm{pH} 5.8$. (Bottom row) Elastic modulus at varying frequencies.

(Figure S3A, Supporting Information). Despite the presence of APTES-coated silica NPs, the elastic modulus of these interfaces is close to zero as can be expected for a clean interface and surface-inactive molecules (Figure S3B, Supporting Information). Figure 5 shows the results of different amounts of ODA in the surrounding oil phase on an aqueous pendant drop with or without NPs. For the lowest concentration of $0.01 \mathrm{mM}$ ODA, the IFT decrease is small with barely any noticeable difference between the three distinct interfaces with minor differences in elastic moduli. At $0.1 \mathrm{mM}$ ODA in the absence of NPs, a plateau is not reached for the studied time scale due to relaxation effects at the interface. ${ }^{44}$ This slower decrease at longer times also occurs for the silica particles associated with a stronger decrease in IFT and equal elasticity values when NPs are absent, showing that the lipid dominates the dilational rheological properties for a silicacovered interface. Contrarily, the adsorption curve with the amine-coated NPs equilibrates within 16 min after the onset of lipid addition and the elastic moduli with these NPs are significantly lower, showing that fewer ODA molecules are present at the interface. More complex behavior is observed at $1 \mathrm{mM}$ ODA as both NP dispersions have IFTs lower than pure water with almost identical elastic moduli for the three cases.

Adsorption of Negatively Charged Silica Nanoparticles in the Presence of a Cationic Amino-Lipid. Highly charged, strongly hydrophilic particles, like silica, can only adsorb at the interface when the energy barrier against adsorption is sufficiently lowered and can be explained in terms of DLVO interactions, mainly due to van der Waals attraction and screening of electrostatic repulsion. ${ }^{72}$ The matching charge between the silica particles and the buried pristine interface is unfavorable as the reflectivity follows the Fresnel line (Figure 2), indicating an adsorbate-free interface, although (brief) adsorption of silica NPs has been previously documented at higher particle concentrations. ${ }^{49,73,74}$ Large amounts of silica NPs at the interface might increase the interfacial roughness due to marginal ordering ${ }^{53}$ or even cause pronounced dips in the XRR profile due to layer formation at the surface. ${ }^{74}$ When ODA is added, the silica NPs in the subsurface region feel the strong attraction of the protonated surfactant head group and form an electrostatic bond between a silanol and the amino group that causes partial charge neutralization along with increased lipophilicity, which forces the particles to pack more closely as observed in Figure 5. ${ }^{44}$ The top view of Figure 6A shows the hexagonal arrangement of the NPs with their contact points below the interface upon dense packing, which is only possible when sufficient double-layer screening occurred. An HCP monolayer is already formed at $0.1 \mathrm{mM}$ as derived from the presence of oscillations and model fitting of the ED. Additionally, the stronger decrease in IFT at longer times, whose shape resembles the adsorption curve in the absence of NPs, gives strong evidence that these surfactantparticle complexes exert a synergistic effect on the IFT due to the formation of a close-packed interfacial film. However, a further increase in surfactant concentration has no effect on the particle coverage or CA at $0.1 \mathrm{w} / \mathrm{w} \% \mathrm{NPs}$, while the IFT continues to show a significant decrease of more than $10 \mathrm{mN}$ $\mathrm{m}^{-1}$. Information about the lateral particle distribution using 

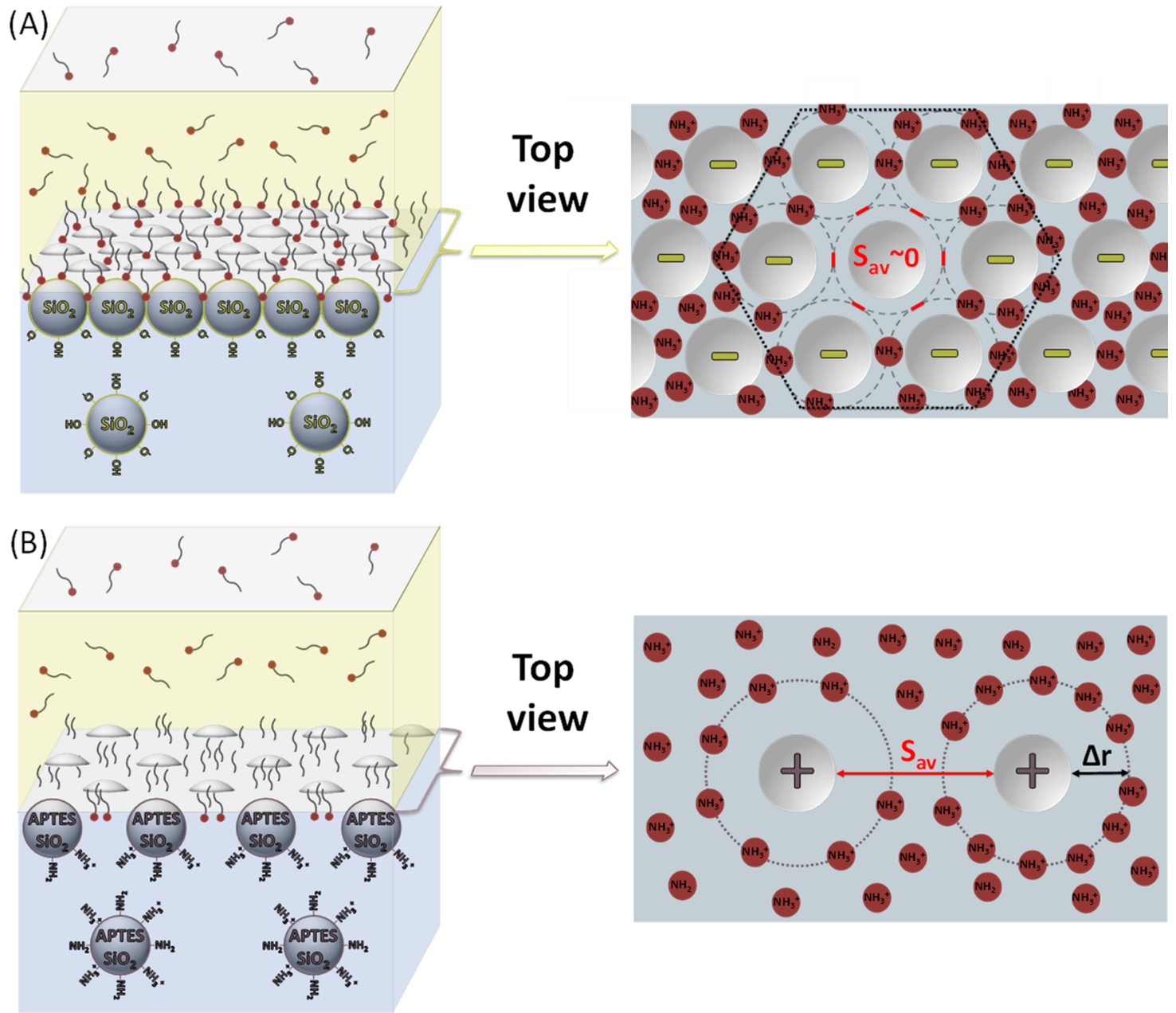

Figure 6. Visual representation of the negatively (A) and positively (B) charged silica nanoparticles adsorbed at the decane-water interface and their interaction with the oil-soluble ODA surfactant molecules at intermediate to high concentrations. In (A), the spreading distance $s_{\mathrm{av}}$ between particles is highlighted (red lines) and adsorbed ODA molecules have been removed for clarity. (A) Negatively charged silica nanoparticles reduce the interfacial energy due to an increase in their CA by interaction with the positively charged amino group of ODA. Local charge neutralization along with increased hydrophobicity of the particles favors hexagonal arrangement (black dotted hexagon) at the interface with a center-to-center distance of one particle diameter (gray striped circles). (B) In the case of positively charged APTES-coated silica nanoparticles, an electrostatic exclusion zone $(\Delta r$, black line) is formed, which rejects the ODA molecules and their packing is only slightly influenced by an increase in ODA concentration, while the exclusion zone becomes smaller as more ODA molecules adsorb.

grazing-incidence small-angle scattering (GISAXS) experiments with the same synchrotron setup could, in principle, confirm if an HCP layer is formed, but in our measurements, the weak off-specular signal was too strongly attenuated by the oil phase. The formation of an adlayer is confirmed by XRR, and the constant particle coverage for 0.1 and $1 \mathrm{mM}$ ODA strongly suggests that these spherical NPs form a densely packed monolayer. This particle monolayer with a CA of roughly $37^{\circ}$ will occupy about $1 / 3$ of the interfacial area, leaving $2 / 3$ of the interface free for surfactants to adsorb. An increase in the effective concentration, where the ionic lipid molecules pack more densely at the interface, explains the observed decrease in IFT at $1 \mathrm{mM}$ ODA without affecting the particle coverage. ${ }^{75}$

Adsorption of Positively Charged Silica Nanoparticles in the Presence of a Cationic Amino-Lipid. When the NPs have a positive charge, they self-assemble at the pristine interface with incomplete coverage (Figure 2). It has been observed that charged colloids trapped at an interface can form highly ordered quasi-crystalline structures through balancing of long- and short-range electrostatic repulsions mediated by the nonpolar medium and asymmetric charge dissociation, respectively, against the long-range attractive interactions due to the deformation of the interface by the electrostatic force induced by its neighboring particles. As the NPs remain mainly dispersed in the aqueous phase, the dipolar interactions mediated through the oil become less significant compared to the electric double-layer repulsion. ${ }^{21,22,48,63,76}$ Despite their adsorption to the pristine oil-water interface, no significant change in IFT is observed owing to their wettability (at HCP coverage without surfactants, the total change in IFT caused by the particles would only be $1.5410^{-4} \mathrm{mN} \mathrm{m}^{-1}$ based on eq 1) and additional methods are required to judge adsorption of hydrophilic NPs (Figure S3C, Supporting information). At $0.01 \mathrm{mM}$ ODA, the IFT and elastic moduli are similar to those of silica NPs, but competition for interfacial coverage is evident from reflectivity data as the lipids desorb some of the initially adsorbed NPs, which can be explained by their small desorption energy of $\sim 19.79 k T$ (Figures 4 and 5). Also, cooperativity between the alkane and low quantities of 
Table 1. Experimental Values for Different Concentrations of ODA with $0.1 \mathrm{w} / \mathrm{w} \%$ Silica Nanoparticles and APTES-Coated Silica Nanoparticles. Contact Angle and Particle Coverage with Their Standard Deviation Were Obtained from the Analysis of the Specular Reflectance Data (XRR) and Calculated from the Analytical Model Based on Equation $3^{46}$ Using the CA Obtained from $\mathrm{XRR}^{a}$

\begin{tabular}{|c|c|c|c|c|c|c|}
\hline \multicolumn{7}{|c|}{ synergistic adsorption $\mathrm{SiO}_{2}$ nanoparticles } \\
\hline conc. $(\mathrm{mM})$ & contact angle XRR ( deg) & coverage XRR $(\varphi)$ & \multicolumn{2}{|c|}{ calculated coverage $(\varphi)$} & \multicolumn{2}{|c|}{ corrected diameter $(\mathrm{nm})$} \\
\hline 0.01 & $34.87 \pm 2.86$ & $0.671 \pm 0.030$ & \multicolumn{2}{|c|}{$0.328 \pm 0.341$} & \multicolumn{2}{|c|}{$15.27 \pm 6.18$} \\
\hline 0.1 & $36.91 \pm 2.25$ & $0.893 \pm 0.012$ & \multicolumn{2}{|c|}{$1.204 \pm 0.469$} & \multicolumn{2}{|c|}{$28.07 \pm 12.16$} \\
\hline 1 & $37.09 \pm 1.31$ & $0.887 \pm 0.026$ & \multicolumn{2}{|c|}{$2.388 \pm 0.533$} & & \pm 20.37 \\
\hline \multicolumn{7}{|c|}{ competitive adsorption APTES-coated $\mathrm{SiO}_{2}$ nanoparticles } \\
\hline conc. $(\mathrm{mM})$ & contact angle XRR (deg) & coverage XRR $(\varphi)$ & erage $(\varphi)$ & corrected & $(\mathrm{nm})$ & $\Delta r(\mathrm{~nm})$ \\
\hline 0.01 & $19.94 \pm 2.90$ & $0.209 \pm 0.019$ & .132 & 34.6 & & $7.89 \pm 1.63$ \\
\hline 0.1 & $27.49 \pm 2.09$ & $0.275 \pm 0.040$ & .266 & 27.2 & & $4.15 \pm 2.75$ \\
\hline 1 & $28.65 \pm 2.13$ & $0.352 \pm 0.024$ & .098 & 16.7 & & $-1.09 \pm 1.50$ \\
\hline
\end{tabular}

${ }^{a}$ In the calculations, the diameter was adjusted until the coverage obtained with XRR was equal to the calculated coverage. $\Delta r$ represents the difference between the measured and the corrected diameter and might relate to the radius of an electrostatic exclusion zone. The standard error for the parameters calculated with eq 3 was obtained from the experimental error of the interfacial tension measurements.

surfactant molecules that form a mixed adsorption layer might enhance particle expulsion in this particular case. ${ }^{77}$ At $0.1 \mathrm{mM}$ ODA, sufficient surfactant is present to monitor IFT changes after particle addition with pendant drop tensiometry. The higher IFT and lower elastic moduli in the presence of APTEScoated NPs compared to the absence of NPs emphasize that a significant portion of surfactant is prevented to adsorb. The slight increase in coverage and CA shows that direct particlesurfactant interactions are strongly inhibited as a result of electrostatic repulsion. This trend in coverage and CA continues with $1 \mathrm{mM}$ ODA as well, but shows a stronger IFT decrease and similar elasticity values to droplets without NPs. This also fits with an increase in effective surfactant concentration as observed for very different IFT values of a densely packed silica monolayer. ${ }^{75}$ Despite requiring sufficiently high lipid concentrations to monitor changes in IFT, the relatively constant particle coverage and CA support that the same competitive mechanism also occurs at lower ODA concentrations.

Interfacial Interaction between Nanoparticles and Cationic Amino-Lipid. It is clear that the charge of the surfactant head group and the NPs either promotes or suppresses particle adsorption. In our previous publication using $80 \mathrm{~nm}$ amine-coated particles, we concluded that a dynamic adsorption/desorption equilibrium exists between the NPs and ODA regulated by the surfactant concentration. By assuming that a difference in equilibrium IFT in the presence and absence of NPs with ODA can only be accounted for by adsorbed NPs, since less space is available for the lipids to adsorb, both their contributions to the IFT were treated as an additive to estimate the particle coverages based on a continuum approach. ${ }^{46}$ Table 1 summarizes the CA and particle coverage obtained from the reflectivity data (XRR) discussed above, as well as the results from the simple analytical model outlined in eq 3 . The calculations for the coverage based on eq 3 use the average IFT measurements (Figure S3C, Supporting Information) and the CA values derived from the XRR data (Figure 4). Even though our model assumes NPs and surfactants are not strongly interacting with each other, as is only the case for APTES-coated silica and ODA, the analysis for silica is also shown for completeness. At first glance, in both cases, the coverages obtained from eq 3 using the average $\mathrm{CA}$ from XRR do not agree well with the scattering data. Since coverage and CA are known from XRR, the coverage was recalculated with varying particle sizes while keeping the other variables constant until both the coverage from XRR and our model matched. This is reported as the corrected diameter in Table 1. With increasing ODA concentrations, the expected diameters of the NPs show opposite behavior that increases in the case of synergistic adsorption and decreases for competitive adsorption. As the model cannot account for particle-surfactant interactions in the synergistic case such as with silica-ODA, no consensus can be formed from these results. This is not the case for the system APTES-coated silica and ODA. Here, due to the repulsive nature between the APTES-coated silica NPs and ODA, the enhanced corrected diameter might correspond to an exclusion zone with radius $\Delta r$, in which the lipids are rejected, similar to the recently reported findings by Papavassilou et al. $^{75}$ The calculated distances for $\Delta r$ are generally smaller than the spreading distance obtained from XRR and therefore fit well in between the particles (Figure 6B). The decrease in $\Delta r$ with increasing surfactant concentration suggests that this exclusion zone is sensitive to the ionic strength at the interface, to which both NPs and surfactants contribute. Inside the exclusion zone, the net repulsive force from the protonated amine groups felt between the NPs and surfactants should be enhanced. ${ }^{11,15,42,43}$

In comparison to our earlier findings with $80 \mathrm{~nm} \mathrm{NPs}$, the contribution of such an exclusion zone might be more pronounced for the interfacial system with the smaller NPs examined in this work. Smaller particles were chosen because of the angular resolution of the XRR diffractometer, which does not allow acceptable resolution of Kiessig fringes of particles $>50 \mathrm{~nm}$. Equation 3, which is simply based on the additivity of all surface energy contributions of the interfacial system, might therefore be only valid if the particle radius is much larger than the radius of the exclusion zone and/or in the presence of moderate concentrations of surfactant at which the exclusion zone is small and the CA of the particles remains unaffected.

\section{CONCLUSIONS}

The adsorption behavior of native negatively charged silica and positively charged APTES-coated silica nanoparticles (NPs) in the presence of an oil-soluble cationic lipid (octadecyl amine, 
ODA) at the decane-water interface was characterized using specular X-ray reflectometry, tensiometry, and dilational rheology. Fitting of the reflectivity curves and subsequent calculation of the electron densities (EDs) using a physically realistic model was compared to a quantitative analysis of the interfacial tension (IFT) based on a continuum approach substantiated with the contact angles (CAs) derived from the scattering experiments to estimate particle coverage. Highresolution in situ X-ray reflectivity data at the pristine airwater surface and oil-water interface showed that silica NPs at $0.1 \mathrm{w} / \mathrm{w} \%$ do not significantly adsorb at the interface contrarily to the APTES-coated silica NPs, which cannot be inferred from oil-water IFT measurements. Bare silica NPs do adsorb at the oil-water interface in the presence of ODA by sufficient surface charge neutralization that forces the NPs to the interface into a hexagonally close-packed (HCP) monolayer with contact angles around $37^{\circ}$, which is consistent with synergistic adsorption. On the other hand, the IFT continues to decrease with increasing ODA concentrations, which stems from the high affinity of the lipids to adsorb at the interface and enhances the effective surfactant concentration between adsorbed NPs. When the charges of the NPs match those of the lipids, competition arises and NPs are being desorbed at low ODA concentrations indicated by a slight increase in coverage $(\sim 21$ to $35 \%)$ and CA $\left(\sim 20\right.$ to $\left.29^{\circ}\right)$. Dilational elasticity for both particle systems is solely dependent on adsorbed ODA, and the deviating behavior when competitive adsorption is at play points to the exclusion of lipid molecules in certain interfacial regions. By introducing an electrostatic exclusion zone around interfacially adsorbed particles in which like-charged surfactants are repelled, the analytical approach to describe the interfacial system as the sum of the individual contributions to interfacial energy ${ }^{46}$ can be validated with the experimental in situ data from X-ray reflectivity. These basic insights underlying the adsorption and interfacial interaction of charged colloids and ionic surfactants have important implications for tailoring particle and surfactant contributions in complex colloidal systems in food, agricultural, cosmetic, and pharmaceutical technologies, as well as in contaminated systems, nonideal particle-stabilized emulsions, and other useful applications.

\section{ASSOCIATED CONTENT}

\section{s) Supporting Information}

The Supporting Information is available free of charge at https://pubs.acs.org/doi/10.1021/acs.langmuir.1c00559.

Ninhydrin calibration curve (Figure S1); schematic representation of the slab model (Figure S2); interfacial tension and dilational rheology in the absence of lipids and surface pressure at different lipid concentrations (Figure S3); schematic setup of X-ray reflectivity at the oil-water interface (Figure S4); X-ray reflectivity cell setup (Figure S5); fitting values from reflectivity fits in Figure 2 (Table S1); and fitting values from reflectivity fits in Figure 3 (Table S2) (PDF)

\section{AUTHOR INFORMATION}

\section{Corresponding Author}

Michael Maas - Advanced Ceramics, University of Bremen, D28359 Bremen, Germany; MAPEX Center for Materials and Processes, University of Bremen, D-28359 Bremen, Germany;
두 orcid.org/0000-0002-2352-4822;

Email: michael.maas@uni-bremen.de

\section{Authors}

Joeri Smits - Advanced Ceramics, University of Bremen, D28359 Bremen, Germany

Rajendra P. Giri - Institute of Experimental and Applied Physics, Kiel University, D-24098 Kiel, Germany

Chen Shen - DESY Photon Science, D-22607 Hamburg, Germany

Diogo Mendonça - Advanced Ceramics, University of Bremen, D-28359 Bremen, Germany; Department of Mechanical Engineering, Federal University of Santa Catarina, 88040-900 Florianopolis, Brazil

Bridget Murphy - Institute of Experimental and Applied Physics and Ruprecht-Haensel Laboratory, Kiel University, D-24098 Kiel, Germany; (1) orcid.org/0000-0002-13542381

Patrick Huber - DESY Photon Science, D-22607 Hamburg, Germany; Institute for Materials and X-Ray Physics, Hamburg University of Technology, 21073 Hamburg, Germany; Center for Hybrid Nanostructures ChyN, Hamburg University, 22607 Hamburg, Germany; (1) orcid.org/0000-0002-2126-9100

Kurosch Rezwan - Advanced Ceramics, University of Bremen, D-28359 Bremen, Germany; MAPEX Center for Materials and Processes, University of Bremen, D-28359 Bremen, Germany; @orcid.org/0000-0002-7318-1119

Complete contact information is available at: https://pubs.acs.org/10.1021/acs.langmuir.1c00559

\section{Author Contributions}

The manuscript was written through contributions of all authors. All authors have given approval to the final version of the manuscript.

\section{Notes}

The authors declare no competing financial interest.

\section{ACKNOWLEDGMENTS}

The authors thank P. Witte for her expertise with the SEM and T. Mehrtens for TEM. They acknowledge DESY (Hamburg, Germany), a member of the Helmholtz Association HGF, for the provision of experimental facilities. Parts of this research were carried out at PETRA III, and the authors thank Dr. M. Lippmann for assistance in using the lab facility. The authors acknowledge research grant Verbundforschung BMBF/ 05KS7FK3/05KS10FK2 and Verbundforschung BMBF/ 05K16FK1 for financing LISA instrument and the Lambda GaAs detector, respectively. M.M. acknowledges support by DFG (Deutsche Forschungsgemeinschaft) project 278836263. P.H. acknowledges support by the DFG Graduate School GRK 2462 "Processes in natural and technical Particle-Fluid-Systems (PintPFS)” (Project number 390794421). This work was also supported by the DFG within the Collaborative Research Initiative SFB 986 "Tailor-Made Multi-Scale Materials Systems” (project number 192346071).

\section{ABBREVIATIONS USED}

APTES, (3-aminopropyl)triethoxysilane; CA, contact angle; $\mathrm{CTAB}$, hexadecyltrimethylammonium bromide; DLVO, Derjaguin-Landau-Verwey-Overbeek; ED, electron density; GISAXS, grazing-incidence small-angle scattering; HCP, 
hexagonal close-packing; IEP, isoelectric point; IFT, interfacial tension; NP, nanoparticle; ODA, octadecyl amine; PDI, polydispersity index; SEM, scanning electron microscopy; SFT, surface tension; SHG, second harmonic generation; TEM, transmission electron microscopy; TEOS, tetraethyl orthosilicate; XRR, X-ray reflectivity

\section{REFERENCES}

(1) Zhang, Y.; Wang, S.; Zhou, J.; Zhao, R.; Benz, G.; Tcheimou, S.; Meredith, J. C.; Behrens, S. H. Interfacial Activity of Nonamphiphilic Particles in Fluid-Fluid Interfaces. Langmuir 2017, 33, 4511-4519.

(2) Bizmark, N.; Ioannidis, M. A.; Henneke, D. E. Irreversible Adsorption-Driven Assembly of Nanoparticles at Fluid Interfaces Revealed by a Dynamic Surface Tension Probe. Langmuir 2014, 30, 710-717.

(3) Du, K.; Glogowski, E.; Emrick, T.; Russell, T. P.; Dinsmore, A. D. Adsorption Energy of Nano- and Microparticles at Liquid-Liquid Interfaces. Langmuir 2010, 26, 12518-12522.

(4) Coertjens, S.; De Dier, R.; Moldenaers, P.; Isa, L.; Vermant, J. Adsorption of Ellipsoidal Particles at Liquid-Liquid Interfaces. Langmuir 2017, 33, 2689-2697.

(5) Ruhland, T. M.; Gröschel, A. H.; Ballard, N.; Skelhon, T. S.; Walther, A.; Müller, A. H. E.; Bon, S. A. F. Influence of Janus Particle Shape on Their Interfacial Behavior at Liquid-Liquid Interfaces. Langmuir 2013, 29, 1388-1394.

(6) Sato, N.; Aoyama, Y.; Yamanaka, J.; Toyotama, A.; Okuzono, T. Particle Adsorption on Hydrogel Surfaces in Aqueous Media Due to van Der Waals Attraction. Sci. Rep. 2017, 7, No. 6099.

(7) Harikrishnan, A. R.; Dhar, P.; Agnihotri, P. K.; Gedupudi, S.; Das, S. K. Effects of Interplay of Nanoparticles, Surfactants and Base Fluid on the Surface Tension of Nanocolloids. Eur. Phys. J. E 2017, 40, 16-23.

(8) Born, P.; Blum, S.; Gerstner, D.; Huber, P.; Kraus, T.; Schön, V. Self-Assembly of Gold Nanoparticles at the Oil - Vapor Interface: From Mono- to Multilayers. Langmuir 2014, 30, 13176-13181.

(9) Ferdous, S.; Ioannidis, M. A.; Henneke, D. E. Effects of Temperature, $\mathrm{PH}$, and Ionic Strength on the Adsorption of Nanoparticles at Liquid-Liquid Interfaces. J. Nanopart. Res. 2012, 14, No. 850.

(10) Manga, M. S.; Hunter, T. N.; Cayre, O. J.; York, D. W.; Reichert, M. D.; Anna, S. L.; Walker, L. M.; Williams, R. A.; Biggs, S. R. Measurements of Submicron Particle Adsorption and Particle Film Elasticity at Oil-Water Interfaces. Langmuir 2016, 32, 4125-4133.

(11) Chai, Y.; Lukito, A.; Jiang, Y.; Ashby, P. D.; Russell, T. P. FineTuning Nanoparticle Packing at Water-Oil Interfaces Using Ionic Strength. Nano Lett. 2017, 17, 6453-6457.

(12) Mysels, K. J.; Florence, A. T. The Effect of Impurities on Dynamic Surface Tension-Basis for a Valid Surface Purity Criterion. J. Colloid Interface Sci. 1973, 43, 577-582.

(13) Jin, J.; Li, X.; Geng, J.; Jing, D. Insights into the Complex Interaction between Hydrophilic Nanoparticles and Ionic Surfactants at the Liquid/Air Interface. Phys. Chem. Chem. Phys. 2018, 20, $15223-15235$.

(14) Putro, J. N.; Ismadji, S.; Gunarto, C.; Soetaredjo, F. E.; Ju, Y. H. A Study of Anionic, Cationic, and Nonionic Surfactants Modified Starch Nanoparticles for Hydrophobic Drug Loading and Release. J. Mol. Liq. 2020, 298, No. 112034.

(15) Kirby, S. M.; Anna, S. L.; Walker, L. M. Effect of Surfactant Tail Length and Ionic Strength on the Interfacial Properties of Nanoparticle-Surfactant Complexes. Soft Matter 2018, 14, 112-123.

(16) Gonzenbach, U. T.; Studart, A. R.; Tervoort, E.; Gauckler, L. J. Tailoring the Microstructure of Particle-Stabilized Wet Foams. Langmuir 2007, 23, 1025-1032.

(17) AlYousef, Z.; Almobarky, M.; Schechter, D. Enhancing the Stability of Foam by the Use of Nanoparticles. Energy Fuels 2017, 31, 10620-10627.

(18) Dreja, M.; Heine, K.; Tieke, B.; Junkers, G. Effects of Functionalized Latex Particles and Anionic Surfactants on the Flow
Behavior of Aqueous Gelatin Dispersions. J. Colloid Interface Sci. 1997, 191, 131-140.

(19) Micheau, C.; Dedovets, D.; Bauduin, P.; Diat, O.; Girard, L. Nanoparticle Foam Flotation for Caesium Decontamination Using a PH-Sensitive Surfactant. Environ. Sci. Nano 2019, 6, 1576-1584.

(20) Pereira, M. L. D. O.; Maia, K. C. B.; Silva, W. C.; Leite, A. C.; Francisco, A. D. D. S.; Vasconcelos, T. L.; Nascimento, R. S. V.; Grasseschi, D. Fe3O4Nanoparticles as Surfactant Carriers for Enhanced Oil Recovery and Scale Prevention. ACS Appl. Nano Mater. 2020, 3, 5762-5772.

(21) Pickering, S. U. Emulsions. J. Chem. Soc. Trans. 1907, 91, 2001-2021.

(22) Ramsden, W. Separation of Solids in the Surface-Layers of Solutions and "Suspensions". Proc. R. Soc. London 1904, 72, 156-164.

(23) Pieranski, P. Two-Dimensional Interfacial Colloidal Crystals. Phys. Rev. Lett. 1980, 45, 569-572.

(24) Levine, S.; Bowen, B. D.; Partridge, S. J. Stabilization of Emulsions by Fine Particles I. Partitioning of Particles between Continuous Phase and Oil/Water Interface. Colloids Surf. 1989, 38, $325-343$.

(25) Binks, B. P. Particles as Surfactants-Similarities and Differences. Curr. Opin. Colloid Interface Sci. 2002, 7, 21-41.

(26) Young, T., III An Essay on the Cohesion of Fluids. Phil. Trans. R. Soc 1805, 95, 65-87.

(27) Law, B. M.; McBride, S. P.; Wang, J. Y.; Wi, H. S.; Paneru, G.; Betelu, S.; Ushijima, B.; Takata, Y.; Flanders, B.; Bresme, F.; Matsubara, H.; Takiue, T.; Aratono, M. Line Tension and Its Influence on Droplets and Particles at Surfaces. Prog. Surf. Sci. 2017, 92, $1-39$.

(28) Dörr, A.; Hardt, S. Line Tension and Reduction of Apparent Contact Angle Associated with Electric Double Layers. Phys. Fluids 2014, 26, No. 082105.

(29) Kutuzov, S.; He, J.; Tangirala, R.; Emrick, T.; Russell, T. P.; Böker, A. On the Kinetics of Nanoparticle Self-Assembly at Liquid/ Liquid Interfaces. Phys. Chem. Chem. Phys. 2007, 9, 6351.

(30) Nelson, A.; Wang, D.; Koynov, K.; Isa, L. A Multiscale Approach to the Adsorption of Core-Shell Nanoparticles at Fluid Interfaces. Soft Matter 2015, 11, 118-129.

(31) Bossa, G. V.; Roth, J.; Bohinc, K.; May, S. The Apparent Charge of Nanoparticles Trapped at a Water Interface. Soft Matter 2016, 12, 4229-4240.

(32) Wu, L.; Wang, X.; Wang, G.; Chen, G. In Situ X-Ray Scattering Observation of Two-Dimensional Interfacial Colloidal Crystallization. Nat. Commun. 2018, 9, No. 1335.

(33) Katiyar, P.; Singh, J. K. A Coarse-Grain Molecular Dynamics Study of Oil-Water Interfaces in the Presence of Silica Nanoparticles and Nonionic Surfactants. J. Chem. Phys. 2017, 146, No. 204702.

(34) Jiménez-Ángeles, F.; Khoshnood, A.; Firoozabadi, A. Molecular Dynamics Simulation of the Adsorption and Aggregation of Ionic Surfactants at Liquid-Solid Interfaces. J. Phys. Chem. C 2017, 121, 25908-25920.

(35) Vatanparast, H.; Javadi, A.; Bahramian, A. Silica Nanoparticles Cationic Surfactants Interaction in Water-Oil System. Colloids Surf., A 2017, 521, 221-230.

(36) Ranatunga, R. J. K. U.; Nguyen, C. T.; Wilson, B. A.; Shinoda, W.; Nielsen, S. O. Molecular Dynamics Study of Nanoparticles and Non-Ionic Surfactant at an Oil-Water Interface. Soft Matter 2011, 7, 6942-6952.

(37) Pichot, R.; Spyropoulos, F.; Norton, I. T. Competitive Adsorption of Surfactants and Hydrophilic Silica Particles at the Oil - Water Interface: Interfacial Tension and Contact Angle Studies. J. Colloid Interface Sci. 2012, 377, 396-405.

(38) Binks, B. P.; Rodrigues, J. A.; Frith, W. J. Synergistic Interaction in Emulsions Stabilized by a Mixture of Silica Nanoparticles and Cationic Surfactant. Langmuir 2007, 23, 3626-3636.

(39) Biswal, N. R.; Rangera, N.; Singh, J. K. Effect of Different Surfactants on the Interfacial Behavior of the N-Hexane-Water System in the Presence of Silica Nanoparticles. J. Phys. Chem. B 2016, 120, $7265-7274$. 
(40) Yazhgur, P. A.; Noskov, B. A.; Liggieri, L.; Lin, S.; Loglio, G.; Miller, R.; Ravera, F. Dynamic Properties of Mixed Nanoparticle/ Surfactant Adsorption Layers. Soft Matter 2013, 9, 3305-3314.

(41) Liggieri, L.; Santini, E.; Guzman, E.; Maestro, A.; Ravera, F. Wide-Frequency Dilational Rheology Investigation of Mixed Silica Nanoparticle - CTAB Interfacial Layers. Soft Matter 2011, 7, 76997709.

(42) Vatanparast, H.; Shahabi, F.; Bahramian, A.; Javadi, A.; Miller, $\mathrm{R}$. The Role of Electrostatic Repulsion on Increasing Surface Activity of Anionic Surfactants in the Presence of Hydrophilic Silica Nanoparticles. Sci. Rep. 2018, 8, No. 7251.

(43) Eftekhari, M.; Schwarzenberger, K.; Javadi, A.; Eckert, K. The Influence of Negatively Charged Silica Nanoparticles on the Surface Properties of Anionic Surfactants: Electrostatic Repulsion or the Effect of Ionic Strength? Phys. Chem. Chem. Phys. 2020, 22, 22382248.

(44) Whitby, C. P.; Fornasiero, D.; Ralston, J.; Liggieri, L.; Ravera, F. Properties of Fatty Amine - Silica Nanoparticle Interfacial Layers at the Hexane - Water Interface. J. Phys. Chem. C 2012, 116, 30503058.

(45) Hua, X.; Bevan, M. A.; Frechette, J. Competitive Adsorption between Nanoparticles and Surface Active Ions for the Oil-Water Interface. Langmuir 2018, 34, 4830-4842.

(46) Smits, J.; Vieira, F.; Bisswurn, B.; Rezwan, K.; Maas, M. Reversible Adsorption of Nanoparticles at Surfactant-Laden LiquidLiquid Interfaces. Langmuir 2019, 35, 11089-11098.

(47) Arnaudov, L. N.; Cayre, O. J.; Cohen Stuart, M. A.; Stoyanov, S. D.; Paunov, V. N. Measuring the Three-Phase Contact Angle of Nanoparticles at Fluid Interfaces. Phys. Chem. Chem. Phys. 2010, 12, 328-331.

(48) Vogel, N.; Ally, J.; Bley, K.; Kappl, M.; Landfester, K.; Weiss, C. K. Direct Visualization of the Interfacial Position of Colloidal Particles and Their Assemblies. Nanoscale 2014, 6, 6879-6885.

(49) Binks, B. P.; Isa, L.; Tyowua, A. T. Direct Measurement of Contact Angles of Silica Particles in Relation to Double Inversion of Pickering Emulsions. Langmuir 2013, 29, 4923-4927.

(50) Zanini, M.; Marschelke, C.; Anachkov, S. E.; Marini, E.; Synytska, A.; Isa, L. Universal Emulsion Stabilization from the Arrested Adsorption of Rough Particles at Liquid-Liquid Interfaces. Nat. Commun. 2017, 8, No. 15701.

(51) Horváth, I. T.; Colinet, P.; Vetrano, M. R. Measuring Contact Angles of Small Spherical Particles at Planar Fluid Interfaces by Light Extinction. Appl. Phys. Lett. 2016, 108, No. 201605.

(52) Wu, W.; Liu, X.; Chen, S. L.; Yuan, Q.; Gan, W. Particle Adsorption at the Oil-Water Interface Studied with Second Harmonic Generation. Soft Matter 2019, 15, 7672-7677.

(53) Calzolari, D. C. E.; Pontoni, D.; Deutsch, M.; Reichert, H.; Daillant, J. Nanoscale Structure of Surfactant-Induced Nanoparticle Monolayers at the Oil-Water Interface. Soft Matter 2012, 8, 1147811483.

(54) Isa, L.; Calzolari, D. C. E.; Pontoni, D.; Gillich, T.; Nelson, A.; Zirbs, R.; Sánchez-Ferrer, A.; Mezzenga, R.; Reimhult, E. Core-Shell Nanoparticle Monolayers at Planar Liquid-Liquid Interfaces: Effects of Polymer Architecture on the Interface Microstructure. Soft Matter 2013, 9, 3789-3797.

(55) Fernández-Toledano, J. C.; Moncho-Jordá, A.; Martínez-López, F.; Hidalgo-Álvarez, R. Spontaneous Formation of Mesostructures in Colloidal Monolayers Trapped at the Air-Water Interface: A Simple Explanation. Langmuir 2004, 20, 6977-6980.

(56) Yadav, A. R.; Sriram, R.; Carter, J. A.; Miller, B. L. Comparative Study of Solution-Phase and Vapor-Phase Deposition of Aminosilanes on Silicon Dioxide Surfaces. Mater. Sci. Eng., C 2014, 35, 283-290.

(57) Ohshima, H. A Simple Expression for Henry's Function for the Retardation Effect in Electrophoresis of Spherical Colloidal Particles. J. Colloid Interface Sci. 1994, 168, 269-271.

(58) Hristov, D. R.; Rocks, L.; Kelly, P. M.; Thomas, S. S.; Pitek, A. S.; Verderio, P.; Mahon, E.; Dawson, K. A. Tuning of Nanoparticle Biological Functionality through Controlled Surface Chemistry and
Characterisation at the Bioconjugated Nanoparticle Surface. Sci. Rep. 2015, No. 17040.

(59) Kimoto, S.; Dick, W. D.; Hunt, B.; Szymanski, W. W.; McMurry, P. H.; Roberts, D. L.; Pui, D. Y. H. Characterization of Nanosized Silica Size Standards. Aerosol Sci. Technol. 2017, 51, 936945.

(60) Goebel, A.; Lunkenheimer, K. Interfacial Tension of the Water/ n-Alkane Interface. Langmuir 1997, 13, 369-372.

(61) Seeck, O. H.; Deiter, C.; Pflaum, K.; Bertam, F.; Beerlink, A.; Franz, H.; Horbach, J.; Schulte-Schrepping, H.; Murphy, B. M.; Greve, M.; Magnussen, O. The High-Resolution Diffraction Beamline P08 at PETRA III. J. Synchrotron Radiat. 2012, 19, 30-38.

(62) Murphy, B. M.; Greve, M.; Runge, B.; Koops, C. T.; Elsen, A.; Stettner, J.; Seeck, O. H.; Magnussen, O. M. A Novel X-Ray Diffractometer for Studies of Liquid-Liquid Interfaces. J. Synchrotron Radiat. 2014, 21, 45-56.

(63) Vignaud, G.; Gibaud, A. REFLEX: A Program for the Analysis of Specular X-Ray and Neutron Reflectivity Data. J. Appl. Crystallogr. 2019, 52, 201-213.

(64) Watanabe, R.; Yokoi, T.; Kobayashi, E.; Otsuka, Y.; Shimojima, A.; Okubo, T.; Tatsumi, T. Extension of Size of Monodisperse Silica Nanospheres and Their Well-Ordered Assembly. J. Colloid Interface Sci. 2011, 360, 1-7.

(65) Avazbaeva, Z.; Sung, W.; Lee, J.; Phan, M. D.; Shin, K.; Vaknin, D.; Kim, D. Origin of the Instability of Octadecylamine Langmuir Monolayer at Low PH. Langmuir 2015, 31, 13753-13758.

(66) Braslau, A.; Pershan, P. S.; Swislow, G.; Ocko, B. M.; AlsNielsen, J. Capillary Waves on the Surface of Simple Liquids Measured by X-Ray Reflectivity. Phys. Rev. A 1988, 38, 2457-2470.

(67) Larson-Smith, K.; Jackson, A.; Pozzo, D. C. SANS and SAXS Analysis of Charged Nanoparticle Adsorption at Oil-Water Interfaces. Langmuir 2012, 28, 2493-2501.

(68) Stillinger, F. H.; Ben-Naim, A. Liquid-Vapor Interface Potential for Water. J. Chem. Phys. 1967, 47, 4431-4437.

(69) Marinova, K. G.; Alargova, R. G.; Denkov, N. D.; Velev, O. D.; Petsev, D. N.; Ivanov, I. B.; Borwankar, R. P. Charging of Oil - Water Interfaces Due to Spontaneous Adsorption of Hydroxyl Ions. Langmuir 1996, 12, 2045-2051.

(70) Beattie, J. K.; Djerdjev, A. M. The Pristine Oil/Water Interface: Surfactant-Free Hydroxide-Charged Emulsions. Angew. Chem., Int. Ed. 2004, 43, 3568-3571.

(71) Vácha, R.; Rick, S. W.; Jungwirth, P.; De Beer, A. G. F.; De Aguiar, H. B.; Samson, J. S.; Roke, S. The Orientation and Charge of Water at the Hydrophobic Oil Droplet - Water Interface. J. Am. Chem. Soc. 2011, 133, 10204-10210.

(72) Dugyala, V. R.; Muthukuru, J. S.; Mani, E.; Basavaraj, M. G. Role of Electrostatic Interactions in the Adsorption Kinetics of Nanoparticles at Fluid-Fluid Interfaces. Phys. Chem. Chem. Phys. 2016, $18,5499-5508$.

(73) Beloqui Redondo, A.; Jordan, I.; Ziazadeh, I.; Kleibert, A.; Giorgi, J. B.; Wörner, H. J.; May, S.; Abbas, Z.; Brown, M. A. Nanoparticle-Induced Charge Redistribution of the Air-Water Interface. J. Phys. Chem. C 2015, 119, 2661-2668.

(74) Madsen, A.; Konovalov, O.; Robert, A.; Grübel, G. Surface Ordering in a Concentrated Suspension of Colloidal Particles Investigated by X-Ray Scattering Methods. Phys. Rev. E 2001, 64, No. 061406.

(75) Vu, T. V.; Papavassiliou, D. V. Synergistic Effects of Surfactants and Heterogeneous Nanoparticles at Oil-Water Interface: Insights from Computations. J. Colloid Interface Sci. 2019, 553, 50-58.

(76) Uppapalli, S.; Zhao, H. The Influence of Particle Size and Residual Charge on Electrostatic Interactions between Charged Colloidal Particles at an Oil-Water Interface. Soft Matter 2014, 10, $4555-4560$.

(77) Fainerman, V. B.; Aksenenko, E. V.; Kovalchuk, V. I.; Mucic, N.; Javadi, A.; Liggieri, L.; Ravera, F.; Loglio, G.; Makievski, A. V.; Schneck, E.; Miller, R. New View of the Adsorption of Surfactants at Water/Alkane Interfaces - Competitive and Cooperative Effects of 
Surfactant and Alkane Molecules. Adv. Colloid Interface Sci. 2020, 279,

No. 102143. 